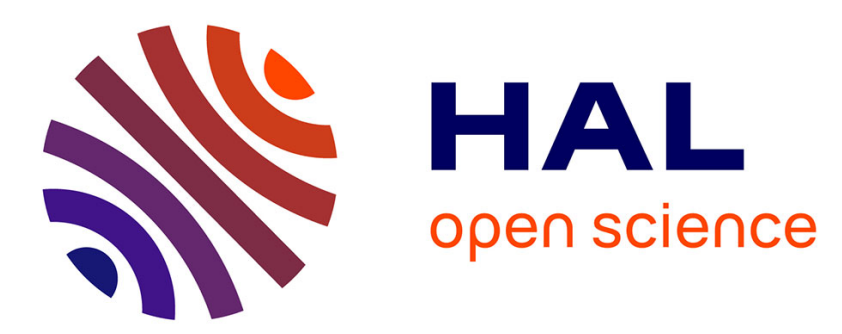

\title{
Linear Stability Analysis of Multiparameter Dynamical Systems via a Numerical-Perturbation Approach
}

\author{
Angelo Luongo, Francesco d'Annibale
}

\section{To cite this version:}

Angelo Luongo, Francesco d'Annibale. Linear Stability Analysis of Multiparameter Dynamical Systems via a Numerical-Perturbation Approach. AIAA Journal, 2011, 49 (9), pp.2047-2056. hal00788090

\section{HAL Id: hal-00788090 \\ https://hal.science/hal-00788090}

Submitted on 13 Feb 2013

HAL is a multi-disciplinary open access archive for the deposit and dissemination of scientific research documents, whether they are published or not. The documents may come from teaching and research institutions in France or abroad, or from public or private research centers.
L'archive ouverte pluridisciplinaire HAL, est destinée au dépôt et à la diffusion de documents scientifiques de niveau recherche, publiés ou non, émanant des établissements d'enseignement et de recherche français ou étrangers, des laboratoires publics ou privés. 


\title{
Linear Stability Analysis of Multiparameter Dynamical Systems via a Numerical-Perturbation Approach
}

\author{
Angelo Luongo* and Francesco D'Annibale ${ }^{\dagger}$ \\ University of L'Aquila, 67100 L'Aquila, Italy
}

\begin{abstract}
A numerical-perturbation method, based on eigenvalue sensitivity analysis, is developed that is able to build up linear stability diagrams of multiparameter dynamical systems. The algorithm furnishes the critical combinations of parameters causing multiple bifurcations of static, dynamical, or mixed types. Moreover, it determines the manifolds in the parameter space on which simple bifurcations take place. Although the method is general, it is illustrated here for codimension-2 bifurcations only, sufficient for highlighting the underlying ideas.
\end{abstract}

\section{Introduction}

D YNAMICAL systems usually depend on parameters. When some of them are varied in some region of the parameter space $\{\boldsymbol{\mu}\} \subseteq \mathbb{R}^{M}$, bifurcations from an equilibrium position are likely to occur. The bifurcations can be both of static (divergence) or dynamic (Hopf) nature, according to the values assumed by the critical eigenvalue(s) of the Jacobian matrix of the system, namely, zero or purely imaginary, respectively. These basic bifurcations occur on smooth codimension 1 surfaces; if the surfaces intersect each other along lines or points, multiple bifurcations occur at these higher codimension geometrical loci [1]. The critical surfaces divide the parameter space in regions in which the system has qualitatively similar behaviors, which are different in each region. The plot of the organized parameter space is called the linear stability diagram, and it gives a comprehensive scenario of the system behavior near the equilibrium. The aim of the linear stability analysis is to build up such a diagram.

When the system to be analyzed has few degrees of freedom, it is not difficult to find the bifurcation loci by manipulating the closed form characteristic polynomial of the Jacobian matrix. The task requires finding the combinations of parameters $\mu_{c}$ such that a number of critical eigenvalues $\lambda_{k}=\lambda_{k}\left(\boldsymbol{\mu}_{c}\right)$ are zero, or have zero real parts, e.g., by applying Routh Hurwitz criterion [1] or related graphical techniques $[2,3]$. In contrast, when the dimension of the system increases, methods that are practical for small dimensional systems become impracticable, and one must resort to pure numerically based analyses.

A pragmatic approach to the problem would consist of using readily available software packages (e.g., AUTO or similar prod ucts). Accordingly, two different strategies are possible. In the first, one should perform, in sequence, the following operations:

1) To draw an equilibrium path (bifurcation diagram) in one parameter, the remaining ones being kept constant.

2) Look for codimension 1 bifurcation points along the path.

3) Start a continuation procedure from these points aimed to build up planar sections of the bifurcation loci in the remaining parameters.

As a drawback, these software have been specifically designed for small dimensional nonlinear systems, as obtained via the center manifold reduction and normal form theory (see, e.g., [4]). When, in contrast, large dimensional linear systems have to be analyzed, one

\footnotetext{
*Professor, Department of Structural Engineering, Water and Land; angelo.luongo@univaq.it.

TPh.D., Department of Structural Engineering, Water and Land;
} francesco.dannibale@univaq.it. should write all the equations in scalar form and (inelegantly) draw a trivial bifurcation diagram $\mathbf{x}=\mathbf{0}$ in one parameter. As a second strategy, also followed by some users, one could 1) expand the (parameter dependent) characteristic polynomial of the Jacobian matrix, leading to two scalar equations in the real and imaginary parts of the eigenvalue, in which the real part must be vanished; 2 ) consider these equations as the right hand members of a fictitious dynamical system, in which two parameters are taken as dummy state variables and the imaginary part of the eigenvalue is taken as a dummy parameter; and 3) look for equilibrium paths for the dummy system, actually representing the bifurcation loci for the real system. If this procedure is employed, some of the bifurcation points found (e.g., Hopf points) could have no meaning for the true system. Although both of these procedures work, they are highly unsatisfactory from a formal point of view, since they are essentially based on tricks; moreover, they are also unsatisfactory from a practical point of view, since they require handling a large number of data (scalar equations or high degree characteristic polynomials).

It therefore seems important to implement a numerical strategy devoted to large dimensional multiparameter linear systems. Purely numerical methods have been applied, mainly based on the Newton method, to solve equations such as $\operatorname{Re}\left[\lambda_{k}(\boldsymbol{\mu})\right]=0$ in the parameter space. These, however, call for numerical evaluations of the derivatives $\partial \lambda_{k}(\boldsymbol{\mu}) / \partial \mu$ of the eigenvalues with respect to the param eters (the so called eigenvalue sensitivities), which require quite expansive reiterate evaluations of the eigenvalues of a large matrix. With the aim being to render the procedure more efficient, one can use a mixed numerical perturbation method, as suggested in $[5,6]$ with reference to codimension 1 bifurcations (saddle node, trans critical, pitchfork, and Hopf bifurcations). Perturbation methods indeed permit the evaluation of the eigenvalue sensitivities in a cheap and easy to implement way, since they just call for solving a chain of linear algebraic equations $[1,7]$. The algorithm just requires the knowledge of the parameter derivatives of the system matrix, which are easy to compute even for large systems, irrespective of whether they are in analytical or numerical form. The procedure illustrated in $[5,6]$ can straightforwardly be extended to higher codimension bifurcations, provided the critical eigenvalues are not coincident. In contrast, if they do coincide, standard perturbation methods fail, since the eigenvalues are not analytical at the coalescence point (in the sense that their first derivatives with respect to the parameters are infinite). In these cases, more sophisticated techniques have to be used to evaluate sensitivities (see $[1,7]$ ).

In this paper, a new method is proposed that exploits the strong potentialities of (enhanced) perturbation methods. The algorithm is able to find, in sequence, 1) the multiple bifurcation loci and 2) the branches of the critical surfaces originating from it, both for non coincident and coincident critical eigenvalues. Reference is made here to a general system undergoing codimension 2 bifurcations. Two bifurcation parameters are therefore sufficient to exhaustively describe the scenario. In Sec. II, the simplest case of noncoincident critical eigenvalues is studied. In Sec. III, the more complex case of 
coincident critical eigenvalues is analyzed. In Sec. IV, some numerical examples are illustrated. Some conclusions are drawn in Sec. V. Finally, Appendix A supplies the background for the pertur bation analysis, Appendix $\underline{B}$ gives some computational details, and Appendices $\underline{\mathrm{C}}$ and $\underline{\mathrm{D}}$ address a related discussion.

\section{Noncoincident Critical Eigenvalues}

Let us consider a finite dimensional dynamical system, admitting the equilibrium position $\mathbf{x}=\mathbf{0}$ for any values of the control param eters $\mu:=\{\mu, \nu\}^{T}$. The motion around the origin is governed by the linear (variational) equation

$$
\dot{\mathbf{x}}=\mathbf{A}(\boldsymbol{\mu}) \mathbf{x}
$$

where $\mathbf{x}$ is the state vector, and $\mathbf{A}(\boldsymbol{\mu})$ is the Jacobian matrix at $\boldsymbol{\mu}$. The goal of the analysis is to find the geometrical loci in the parameter $\boldsymbol{\mu}$ plane at which $\mathbf{A}(\boldsymbol{\mu})$ admits one or two eigenvalues with zero real parts. Here, a bifurcation of codimension 1 or 2 takes place.

\section{A. Eigenvalue Sensitivity}

Let us assume first that the Jacobian matrix $\mathbf{A}=\mathbf{A}(\boldsymbol{\mu})$ of the system admits two distinct critical eigenvalues at the (unknown) bifurcation point $C$. In [7], it was shown that, given a matrix $\mathbf{A}=\mathbf{A}(\boldsymbol{\mu})$, the following series expansion holds for its eigenvalues $\lambda_{k}(\boldsymbol{\mu})$ (see Appendix $\underline{\mathrm{A}}$ ):

$$
\begin{aligned}
& \left(\begin{array}{l}
\lambda_{1}(\boldsymbol{\mu}+\delta \boldsymbol{\mu}) \\
\lambda_{2}(\boldsymbol{\mu}+\delta \boldsymbol{\mu})
\end{array}\right)=\left(\begin{array}{l}
\lambda_{1}(\boldsymbol{\mu}) \\
\lambda_{2}(\boldsymbol{\mu})
\end{array}\right)+\left[\begin{array}{ll}
S_{1 \mu}(\boldsymbol{\mu}) & S_{1 v}(\boldsymbol{\mu}) \\
S_{2 \mu}(\boldsymbol{\mu}) & S_{2 v}(\boldsymbol{\mu})
\end{array}\right]\left(\begin{array}{c}
\delta \mu \\
\delta v
\end{array}\right) \\
& \quad+O\left(|\delta \boldsymbol{\mu}|^{2}\right)
\end{aligned}
$$

in which $S_{k \alpha}(\boldsymbol{\mu})(k=1,2 ; \alpha=\mu, v)$ are the eigenvalue sensitivities at $\boldsymbol{\mu}$, given by

$$
\left[S_{k \alpha}(\boldsymbol{\mu})\right]:=\left[\begin{array}{ll}
\mathbf{y}_{1}^{H}(\boldsymbol{\mu}) \mathbf{A}_{\mu}(\boldsymbol{\mu}) \mathbf{x}_{1}(\boldsymbol{\mu}) & \mathbf{y}_{1}^{H}(\boldsymbol{\mu}) \mathbf{A}_{v}(\boldsymbol{\mu}) \mathbf{x}_{1}(\boldsymbol{\mu}) \\
\mathbf{y}_{2}^{H}(\boldsymbol{\mu}) \mathbf{A}_{\mu}(\boldsymbol{\mu}) \mathbf{x}_{2}(\boldsymbol{\mu}) & \mathbf{y}_{2}^{H}(\boldsymbol{\mu}) \mathbf{A}_{v}(\boldsymbol{\mu}) \mathbf{x}_{2}(\boldsymbol{\mu})
\end{array}\right]
$$

and where $\mathbf{A}_{\mu}:=\partial \mathbf{A} / \partial \mu, \mathbf{A}_{v}:=\partial \mathbf{A} / \partial \nu$, the apex $H$ denotes the transpose conjugate and, moreover, $\mathbf{x}_{k}(\boldsymbol{\mu})$ and $\mathbf{y}_{k}(\boldsymbol{\mu})$ are the right and left eigenvectors of $\mathbf{A}(\boldsymbol{\mu})$, respectively, associated with $\lambda_{k}(\boldsymbol{\mu})$.

\section{B. Searching for the Critical Point}

The search for the (unknown) critical point $C$, at which $\operatorname{Re}\left(\lambda_{k}\left(\boldsymbol{\mu}_{c}\right)\right)=0(k=1,2)$ (dynamic bifurcation), is performed through an iterative scheme (Newton method) based on the linear extrapolation of the eigenvalues. If an approximation $\boldsymbol{\mu}_{i}=\left\{\mu_{i}, v_{i}\right\}^{T}$ of $\boldsymbol{\mu}_{c}$ is known, we look for parameter increments $\delta \boldsymbol{\mu}:=\boldsymbol{\mu}_{i+1}-\boldsymbol{\mu}_{i}$ such that $\operatorname{Re}\left(\lambda_{k}(\boldsymbol{\mu}+\delta \boldsymbol{\mu})\right)=0$; from Eq. (2), by neglecting the reminder, it follows that

$$
\left[\begin{array}{ll}
\operatorname{Re}\left(S_{1 \mu}\left(\boldsymbol{\mu}_{i}\right)\right) & \operatorname{Re}\left(S_{1 v}\left(\boldsymbol{\mu}_{i}\right)\right) \\
\operatorname{Re}\left(S_{2 \mu}\left(\boldsymbol{\mu}_{i}\right)\right) & \operatorname{Re}\left(S_{2 v}\left(\boldsymbol{\mu}_{i}\right)\right)
\end{array}\right]\left(\begin{array}{c}
\mu_{i+1}-\mu_{i} \\
v_{i+1}-v_{i}
\end{array}\right)=-\left(\begin{array}{l}
\operatorname{Re}\left(\lambda_{1}\left(\boldsymbol{\mu}_{i}\right)\right) \\
\operatorname{Re}\left(\lambda_{2}\left(\boldsymbol{\mu}_{i}\right)\right)
\end{array}\right)
$$

If, in contrast, the bifurcation is of mixed static dynamic type, the $\mathrm{Re}$ operator must be omitted in a row (the double zero case will be analyzed later in the paper). It should be noted that Eq. (4) does not require numerical evaluations of the sensitivities via incremental ratios, as usually done in purely numerical methods, since these are furnished by the perturbation analysis [Eq. (3)].

By resuming, the algorithm is as follows:

1) Evaluate (e.g., by a standard QR algorithm) the two eigenvalues of matrix $\mathbf{A}=\mathbf{A}\left(\boldsymbol{\mu}_{i}\right)$ with the smallest real parts, likely to become critical (e.g., one real and the other complex or two complex not conjugate), with the associated right and left eigenvectors $\mathbf{x}_{k}\left(\boldsymbol{\mu}_{i}\right)$, $\mathbf{y}_{k}\left(\boldsymbol{\mu}_{i}\right)$

2) Compute, via Eq. (3), the four sensitivities $S_{k \alpha}\left(\boldsymbol{\mu}_{i}\right)$;

3) Solve the linear system [Eq. (4)] for the $i+1$ approximation of the parameter vector $\boldsymbol{\mu}_{i+1}=\left\{\mu_{i+1}, v_{i+1}\right\}^{T}$;
4) If $\left|\boldsymbol{\mu}_{i+1}-\boldsymbol{\mu}_{i}\right|>$ toll $_{1}$ and $\left|\operatorname{Re}\left(\lambda_{k}\left(\boldsymbol{\mu}_{i+1}\right)\right)\right|>\operatorname{toll}_{2}(k=1,2)$, then execute a new iteration with $i=i+1$; if, instead, $\mid \boldsymbol{\mu}_{i+1}-$ $\boldsymbol{\mu}_{i} \mid \leq$ toll $_{1}$ or $\left|\operatorname{Re}\left(\lambda_{k}\left(\boldsymbol{\mu}_{i+1}\right)\right)\right| \leq \operatorname{toll}_{2} \quad(k=1,2)$, then assume $\boldsymbol{\mu}_{c}=\boldsymbol{\mu}_{i+1}$.

\section{Building Up Bifurcation Loci}

Once the critical point $C$ has been determined in the parameter plane, the two curves originating from it, which are loci of simple bifurcations, are sought for. On each of them, just one eigenvalue $\left(\lambda_{1}\right.$ or $\lambda_{2}$, respectively) is critical, the other having a nonzero real part; consequently, the relevant equation is $\operatorname{Re}\left(\lambda_{k}(\mu, \nu)\right)=0$ (or $\left.\lambda_{k}(\mu, \nu)=0\right)$ for $k=1$ or $k=2$. This equation implicitly defines a curve in the $(\mu, v)$ plane, passing through $C$. After linearization around a point $\boldsymbol{\mu}_{i}=\left\{\mu_{i}, v_{i}\right\}^{T}$, and according to Eq. (2), it reads

$$
\begin{gathered}
\operatorname{Re}\left(S_{k \mu}\left(\boldsymbol{\mu}_{i}\right)\right)\left(\mu_{i+1}-\mu_{i}\right)+\operatorname{Re}\left(S_{k v}\left(\boldsymbol{\mu}_{i}\right)\right)\left(v_{i+1}-v_{i}\right) \\
\quad=-\operatorname{Re}\left(\lambda_{k}\left(\boldsymbol{\mu}_{i}\right)\right) \quad k=1 \quad \text { or } 2
\end{gathered}
$$

An iterative scheme could directly be applied to Eq. (5) in order to obtain a Cartesian representation for the curve of the form $\mu=\mu(\nu)$ or $v=v(\mu)$; however, as is well known, such a representation fails at the turning points. Therefore, a parametric representation of the curve [namely, $\mu=\mu(s), v=v(s)$ with $s$ a parameter] is preferable. To obtain it, a (constraint) scalar equation must be appended to Eq. (5) in order to define the meaning of $s$; the more common choices for the constraint are referred to in literature as the arclength method or the pseudoarclength method [8] (Fig. 1). When a point $\mu_{0}$ is known on the curve [i.e., $\operatorname{Re}\left(\lambda_{k}\left(\bar{\mu}_{0}\right)\right)=0$ ], a close point is sought iteratively as $\boldsymbol{\mu}_{i}, \boldsymbol{\mu}_{i+1}, \ldots$. According to the arclength method, $\left|\boldsymbol{\mu}_{i+1}-\boldsymbol{\mu}_{0}\right|=|\Delta s|$ is fixed for some small increment $|\Delta s|$ of the modulus of the parameter (with $\Delta s>0$ or $\Delta s<0$ ), and the following constraint equation is appended to Eq. (5):

$$
\sqrt{\left(\mu_{i+1}-\mu_{0}\right)^{2}+\left(v_{i+1}-v_{0}\right)^{2}}=|\Delta s|
$$

According to the pseudoarclength method, $\left(\boldsymbol{\mu}_{i+1}-\boldsymbol{\mu}_{0}\right) \cdot \mathbf{t}_{k 0}=\Delta s$ is instead prescribed, namely,

$$
a_{k 0}\left(\mu_{i+1}-\mu_{0}\right)+b_{k 0}\left(v_{i+1}-v_{0}\right)=\Delta s
$$

where $\mathbf{t}_{k 0}=\left\{a_{k 0}, b_{k 0}\right\}^{T}$ is the unit vector tangent to the curve $(k=1$ or 2) at $\boldsymbol{\mu}_{0}$ and, moreover,

$$
\begin{array}{ll}
a_{k 0}:=+\frac{\operatorname{Re}\left(S_{k v}\left(\boldsymbol{\mu}_{0}\right)\right)}{\sqrt{\left[\operatorname{Re}\left(S_{k \mu}\left(\boldsymbol{\mu}_{0}\right)\right)\right]^{2}+\left[\operatorname{Re}\left(S_{k v}\left(\boldsymbol{\mu}_{0}\right)\right)\right]^{2}}}, & k=1 \quad \text { or } 2 \\
b_{k 0}:=-\frac{\operatorname{Re}\left(S_{k \mu}\left(\boldsymbol{\mu}_{0}\right)\right)}{\sqrt{\left[\operatorname{Re}\left(S_{k \mu}\left(\boldsymbol{\mu}_{0}\right)\right)\right]^{2}+\left[\operatorname{Re}\left(S_{k v}\left(\boldsymbol{\mu}_{0}\right)\right)\right]^{2}}}, & k=1 \text { or } 2
\end{array}
$$

are its components. Thus, in the two approaches, $\boldsymbol{\mu}_{i+1}$ moves on a circle of radius $|\Delta s|$ centered at $\mu_{0}$, or along a line that is parallel to

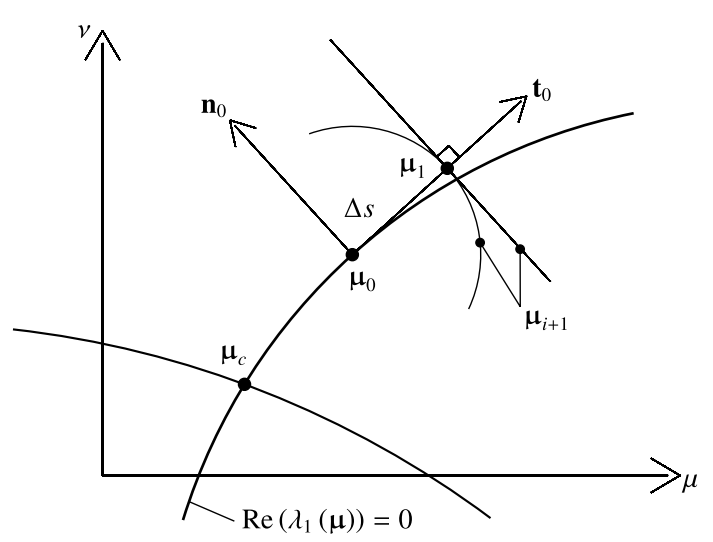

Fig. 1 Arclength and pseudoarclength iterative methods. 
the normal $\mathbf{n}_{k 0}$ to the curve at $\boldsymbol{\mu}_{0}$, at a distance $|\Delta s|$ from it (Fig. 1). In both cases, the ambiguity of the sign of $\Delta s$ refers to the two opposite directions in which the curve can be traveled.

The constraint equation (6) is nonlinear, whereas the constraint equation (7) is linear. To keep the whole problem linear, the pseudoarclength method is adopted here. The relevant algorithm is detailed as follows:

1) Take the critical point $\boldsymbol{\mu}_{c}$ as initial point $\boldsymbol{\mu}_{0}$ and evaluate the sensitivities $S_{k \mu}\left(\boldsymbol{\mu}_{0}\right)$ and $S_{k v}\left(\boldsymbol{\mu}_{0}\right)$ via Eq. (3); then, compute the direction cosines $a_{k 0}$ and $b_{k 0}$ [Eq. (8)];

2) Select a new point $\boldsymbol{\mu}_{1}$ (predictor phase) on the tangent $\mathbf{t}_{k 0}=$ $\left\{a_{k 0}, b_{k 0}\right\}^{T}$ at $\mu_{0}$ at a sufficiently small distance $|\Delta s|$ from $\boldsymbol{\mu}_{0}$, having coordinates

$$
\mu_{1}=\mu_{0}+a_{k} \Delta s, \quad v_{1}=v_{0}+b_{k} \Delta s, \quad k=1 \quad \text { or } 2
$$

3) Solve iteratively (for $i=1,2, \ldots$ ) the following equations in the unknown $\mu_{i+1}, v_{i+1}$ (corrector phase):

$$
\left(\begin{array}{cc}
\operatorname{Re}\left(S_{k \mu}\left(\boldsymbol{\mu}_{i}\right)\right) & \operatorname{Re}\left(S_{k v}\left(\boldsymbol{\mu}_{i}\right)\right) \\
a_{k 0} & b_{k 0}
\end{array}\right)\left(\begin{array}{c}
\mu_{i+1}-\mu_{i} \\
v_{i+1}-v_{i}
\end{array}\right)=\left(\begin{array}{c}
-\operatorname{Re}\left(\lambda_{k}\left(\boldsymbol{\mu}_{i}\right)\right) \\
\Delta s
\end{array}\right)
$$

4) If $\left|\boldsymbol{\mu}_{i+1}-\boldsymbol{\mu}_{i}\right|>\operatorname{toll}_{1}$ and $\left|\operatorname{Re}\left(\lambda_{k}\left(\boldsymbol{\mu}_{i+1}\right)\right)\right|>\operatorname{toll}_{2}(k=1$ or 2$)$, then execute a new iteration [Eq. (10)]; if, instead, $\left|\boldsymbol{\mu}_{i+1}-\boldsymbol{\mu}_{i}\right| \leq$ toll $_{1}$ or $\left|\operatorname{Re}\left(\lambda_{k}\left(\mu_{i+1}\right)\right)\right| \leq \operatorname{toll}_{2}(k=1$ or 2$)$, then assume as a new point $\boldsymbol{\mu}_{0}=\boldsymbol{\mu}_{i+1}$, and restart from step 2 .

\section{Coincident Critical Eigenvalues}

\section{A. Eigenvalue Sensitivity}

The eigenvalue sensitivity analysis for a Jacobian matrix $\mathbf{A}(\boldsymbol{\mu})$ is more difficult when several eigenvalues coincide at the multiple bifurcation point $C$, being all zero (multiple zero bifurcation) or equal to the same pair of complex conjugate purely imaginary numbers (multiple Hopf bifurcation). In these cases, $\mathbf{A}\left(\boldsymbol{\mu}_{c}\right)$ is (generally) defective at the bifurcation; that is, it does not posses a complete set of eigenvalues. Consequently, it is nearly defective close to the critical point; that is, a complete set of eigenvectors does exist, but some of them are nearly coincident. It was shown in [9] that sensitivities of nearly defective eigenvalues cannot be evaluated independently, as in Eq. (2), but they are, in contrast, coupled. Moreover, the nonana lytical nature of the eigenvalues requires using fractional power expansions in the parameters.

Here, we limit ourselves to the simplest case of two real critical eigenvalues (double zero, or Takens Bogdanov, bifurcation), which is generic in two parameter families of systems. As shown in [9], the problem of two nearly coincident eigenvalues $\lambda_{1}(\mu) \simeq \lambda_{2}(\mu)$ of a nearly defective matrix $\mathbf{A}(\boldsymbol{\mu})$ is overcome by starting the expansion, not from the actual system, but by an ideal system $\mathbf{A}_{0}(\boldsymbol{\mu} ; \xi(\boldsymbol{\mu}))$ belonging to an enlarged parameter space $\{\boldsymbol{\mu}, \xi\}$ in which the two eigenvalues coalesce at $\lambda_{0}(\boldsymbol{\mu}):=\left(\lambda_{1}(\boldsymbol{\mu})+\lambda_{2}(\boldsymbol{\mu})\right) / 2$. To achieve this goal, an inverse problem must be solved in which the small additional parameter $\xi \in \mathbb{R}$ must be determined in order to render $\mathbf{A}_{0}(\boldsymbol{\mu} ; \xi(\boldsymbol{\mu}))$ defective. After that, the sensitivities of $\lambda_{0}(\boldsymbol{\mu})$ must be evaluated. is

According to [9] (see also Appendix A), the ideal defective matrix

$$
\mathbf{A}_{0}(\boldsymbol{\mu} ; \xi(\boldsymbol{\mu}))=\mathbf{A}(\boldsymbol{\mu})-\xi(\boldsymbol{\mu}) \mathbf{x}_{20}(\boldsymbol{\mu}) \mathbf{y}_{10}^{H}(\boldsymbol{\mu})
$$

where

$$
\begin{gathered}
\xi(\boldsymbol{\mu})=\frac{1}{4}\left[\lambda_{1}(\boldsymbol{\mu})-\lambda_{2}(\boldsymbol{\mu})\right]^{2} \\
\mathbf{x}_{20}(\boldsymbol{\mu})=\frac{1}{2}\left(\mathbf{x}_{1}(\boldsymbol{\mu})-\mathbf{x}_{2}(\boldsymbol{\mu})\right), \quad \mathbf{y}_{10}(\boldsymbol{\mu})=\xi^{-1 / 2}\left(\mathbf{y}_{1}(\boldsymbol{\mu})+\mathbf{y}_{2}(\boldsymbol{\mu})\right) \\
\mathbf{x}_{10}(\boldsymbol{\mu})=\frac{1}{2} \xi^{1 / 2}\left(\mathbf{x}_{1}(\boldsymbol{\mu})+\mathbf{x}_{2}(\boldsymbol{\mu})\right), \quad \mathbf{y}_{20}(\boldsymbol{\mu})=\mathbf{y}_{1}(\boldsymbol{\mu})-\mathbf{y}_{2}(\boldsymbol{\mu})
\end{gathered}
$$

are the additional perturbation parameter $\xi$ and the generalized right and left eigenvectors $\left(\mathbf{x}_{20}, \mathbf{y}_{10}\right)$ of $\mathbf{A}_{0}(\boldsymbol{\mu} ; \xi(\boldsymbol{\mu}))$, respectively; the proper right and left eigenvectors $\left(\mathbf{x}_{10}, \mathbf{y}_{20}\right)$ of the same matrix will be used later. All these quantities are evaluated from the (nearly coincident) eigenvalues $\lambda_{k}$ and associated right and left eigenvectors, $\mathbf{x}_{k}$ and $\mathbf{y}_{k}$, of the given matrix $\mathbf{A}(\boldsymbol{\mu})$. It should be noticed that, if the eigenvalues $\lambda_{k}$ are complex conjugate, than $\mathbf{x}_{10}$ and $\mathbf{y}_{10}$ are real, while $\mathbf{x}_{20}$ and $\mathbf{y}_{20}$ are purely imaginary; based on this, it is easy to check that all the quantities involved in the following analysis are real.

Second order sensitivity analysis of $\mathbf{A}_{0}(\boldsymbol{\mu} ; \xi(\boldsymbol{\mu}))$, carried out along the lines of [9], leads (after some manipulations) to the following second degree sensitivity equation in the increment $\Delta \lambda_{1,2}:=\lambda_{1,2}(\boldsymbol{\mu}+\delta \boldsymbol{\mu})-\lambda_{0}(\boldsymbol{\mu}):$

$$
\begin{aligned}
& \Delta \lambda^{2}-\left(S_{1 \mu}(\boldsymbol{\mu}) \delta \mu+S_{1 v}(\boldsymbol{\mu}) \delta v\right) \Delta \lambda-\left(\xi(\boldsymbol{\mu})+S_{2 \mu}(\boldsymbol{\mu}) \delta \mu\right. \\
& \left.\quad+S_{2 v}(\boldsymbol{\mu}) \delta v\right)+\mathcal{O}\left(|\delta \boldsymbol{\mu}|^{3 / 2}\right)=0
\end{aligned}
$$

where

$$
\begin{aligned}
& S_{1 \alpha}(\boldsymbol{\mu}):=\mathbf{y}_{20}^{H}(\boldsymbol{\mu}) \mathbf{A}_{\alpha}(\boldsymbol{\mu}) \mathbf{x}_{20}(\boldsymbol{\mu})-\mathbf{y}_{20}^{H}(\boldsymbol{\mu}) \mathbf{u}_{\alpha}^{*}(\boldsymbol{\mu}) \\
& S_{2 \alpha}(\boldsymbol{\mu}):=\mathbf{y}_{20}^{H}(\boldsymbol{\mu}) \mathbf{A}_{\alpha}(\boldsymbol{\mu}) \mathbf{x}_{10}(\boldsymbol{\mu}), \quad \alpha=\mu, v
\end{aligned}
$$

are called sensitivities of order 1 and order $\frac{1}{2}$, respectively and, moreover, $\mathbf{u}_{\mu}^{*}(\boldsymbol{\mu})$ and $\mathbf{u}_{v}^{*}(\boldsymbol{\mu})$ are solutions for the following linear problems:

$$
\left\{\begin{array}{l}
{\left[\mathbf{A}_{0}(\boldsymbol{\mu})-\lambda_{0}(\boldsymbol{\mu}) \mathbf{I}\right] \mathbf{u}_{\alpha}^{*}(\boldsymbol{\mu})=S_{2 \alpha} \mathbf{x}_{20}(\boldsymbol{\mu})-\mathbf{A}_{\alpha}(\boldsymbol{\mu}) \mathbf{x}_{10}(\boldsymbol{\mu})} \\
\mathbf{e}_{h}^{T} \mathbf{u}_{\alpha}^{*}(\boldsymbol{\mu})=0, \quad \alpha=\mu, v
\end{array}\right.
$$

made unique by a normalization condition (here, $\mathbf{e}_{h}$ is the $h$ th $N$ dimensional canonical vector). Note that, when $\delta \mu=\delta \nu=0$, Eq. (13) correctly leads to $\lambda_{1}$ and $\lambda_{2}$. Therefore, $\xi(\mu)$ brings back from the ideal $\mathbf{A}_{0}(\boldsymbol{\mu} ; \xi(\boldsymbol{\mu}))$ to the actual system $\mathbf{A}(\boldsymbol{\mu})$, while $\delta \mu$ and $\delta v$ account for the true perturbation; the two effects, however, cannot be separated.

Equation (13) shows that $\Delta \lambda=\mathcal{O}\left((\xi+|\Delta \mu|)^{1 / 2}\right)$. If $\xi=0$ (i.e., $\left.\boldsymbol{\mu}=\boldsymbol{\mu}_{c}\right)$, then $\Delta \lambda / \Delta \boldsymbol{\mu} \rightarrow \infty$ when $\Delta \boldsymbol{\mu} \rightarrow 0$, this denoting that $\lambda\left(\mu_{c}\right)$ is not analytical at the coalescence point. The increment $\Delta \lambda$ is therefore mainly governed by sensitivities of order $\frac{1}{2} S_{2 \alpha}$; however, there always exists a special combination of the increments of the parameters $\delta \mu$ (i.e., a singular direction in the parameter space) for which $S_{2 \alpha}=0$, this entailing that $\Delta \lambda=\mathcal{O}(|\Delta \mu|)$ in a narrow angular sector containing this direction.

\section{B. Searching for the Critical Point}

Let us assume to know a trial set of parameters $\boldsymbol{\mu}_{i}$, close to $\boldsymbol{\mu}_{c}$, for which the two critical conditions $\lambda_{1,2}\left(\boldsymbol{\mu}_{c}\right)=0$ are approximately satisfied. To refine the approximation, we can use the sensitivity equation (13) (with the remainder neglected), which furnishes, with $\boldsymbol{\mu}=\boldsymbol{\mu}_{i}$ and $\delta \boldsymbol{\mu}=\boldsymbol{\mu}_{i+1}-\boldsymbol{\mu}_{i}$, the eigenvalues $\lambda_{1,2}\left(\boldsymbol{\mu}_{i+1}\right)=$ $\lambda_{0}\left(\boldsymbol{\mu}_{i}\right)+\Delta \lambda_{1,2}\left(\boldsymbol{\mu}_{i}, \delta \boldsymbol{\mu}\right)$; this guides us in choosing the increment $\delta \boldsymbol{\mu}$ making $\lambda_{1,2}\left(\boldsymbol{\mu}_{i+1}\right)=0$. The operation is easily carried out if we rewrite the sensitivity equation $(13)$ in the form of a reduced characteristic equation:

$$
\lambda^{2}-I_{1}(\boldsymbol{\mu}, \delta \boldsymbol{\mu}) \lambda-I_{2}(\boldsymbol{\mu}, \delta \boldsymbol{\mu})+\mathcal{O}\left(|\delta \mu|^{3 / 2}\right)=0
$$

where

$$
\begin{aligned}
& I_{1}(\boldsymbol{\mu}, \delta \boldsymbol{\mu}):=S_{1 \mu}(\boldsymbol{\mu}) \delta \mu+S_{1 v}(\boldsymbol{\mu}) \delta v+2 \lambda_{0}(\boldsymbol{\mu}) \\
& I_{2}(\boldsymbol{\mu}, \delta \boldsymbol{\mu}):=S_{2 \mu}(\boldsymbol{\mu}) \delta \mu+S_{2 v}(\boldsymbol{\mu}) \delta v-\lambda_{0}(\boldsymbol{\mu}) I_{1}(\boldsymbol{\mu}, \delta \boldsymbol{\mu}) \\
& \quad+\xi(\boldsymbol{\mu})+\lambda_{0}^{2}(\boldsymbol{\mu})
\end{aligned}
$$

and we require the invariants to vanish simultaneously, namely, $I_{1}\left(\boldsymbol{\mu}_{i}, \delta \boldsymbol{\mu}\right)=0$ and $I_{2}\left(\boldsymbol{\mu}_{i}, \delta \boldsymbol{\mu}\right)=0$; that is,

$$
\left[\begin{array}{ll}
S_{1 \mu}\left(\boldsymbol{\mu}_{i}\right) & S_{1 v}\left(\boldsymbol{\mu}_{i}\right) \\
S_{2 \mu}\left(\boldsymbol{\mu}_{i}\right) & S_{2 v}\left(\boldsymbol{\mu}_{i}\right)
\end{array}\right]\left(\begin{array}{c}
\mu_{i+1}-\mu_{i} \\
v_{i+1}-v_{i}
\end{array}\right)=-\left(\begin{array}{c}
\lambda_{1}(\boldsymbol{\mu})+\lambda_{2}(\boldsymbol{\mu}) \\
\left(\lambda_{1}^{2}(\boldsymbol{\mu})+\lambda_{2}^{2}(\boldsymbol{\mu})\right) / 2
\end{array}\right)
$$

From these equations, an enhanced approximation for the critical parameters, $\boldsymbol{\mu}_{i+1}=\left\{\mu_{i+1}, v_{i+1}\right\}^{T}$, is drawn, and the procedure can 
be reiterated up to the desired tolerance. It is worth stressing that, while the invariants are nonlinear in $\boldsymbol{\mu}$, they are linear in the increments $\delta \boldsymbol{\mu}$, so that in the iterative approach, Eq. (18) still appears in the linear form, as in the nondefective case [Eq. (4)]

By summarizing, the $i$ th iteration of the algorithm is as follows:

1) Evaluate (e.g., by a standard QR algorithm) the two eigenvalues of matrix $\mathbf{A}=\mathbf{A}\left(\boldsymbol{\mu}_{i}\right)$ having the smallest real parts (both real or complex conjugate) and the associated right and left eigenvectors;

2) Compute the quantities in Eqs. (11) and (12);

3) Calculate $\mathbf{u}_{\mu}^{*}(\boldsymbol{\mu}), \mathbf{u}_{v}^{*}(\boldsymbol{\mu})$ by using Eq. (15) and the sensitivity coefficients in Eq. (14);

4) Solve Eq. (18) for the new parameter set $\boldsymbol{\mu}_{i+1}=\left\{\mu_{i+1}, \nu_{i+1}\right\}^{T}$;

5) If $\left|\boldsymbol{\mu}_{i+1}-\overline{\boldsymbol{\mu}}_{i}\right|>$ toll $_{1}$ and $\left|\operatorname{Re}\left(\lambda_{k}\left(\boldsymbol{\mu}_{i+1}\right)\right)\right|>\operatorname{toll}_{2}(k=1,2)$, then execute a new iteration with $i=i+1$; if, instead, $\mid \boldsymbol{\mu}_{i+1}-$ $\boldsymbol{\mu}_{i} \mid \leq$ toll $_{1}$ or $\left|\operatorname{Re}\left(\lambda_{k}\left(\boldsymbol{\mu}_{i+1}\right)\right)\right| \leq$ toll $_{2} \quad(k=1,2)$, then assume $\boldsymbol{\mu}_{c}=\boldsymbol{\mu}_{i+1}$.

\section{Building Up the Bifurcation Loci}

After having determined the critical point $C$, the construction of the critical manifolds must be tackled. The two invariants in Eq. (17), evaluated at $\boldsymbol{\mu}=\boldsymbol{\mu}_{c}$ and equated to zero, provide the equations of the tangents to the two loci at the critical point: namely, $I_{2}\left(\boldsymbol{\mu}_{c}, \delta \boldsymbol{\mu}\right)=0$ is the (straight line) tangent to the divergence locus, while $I_{1}\left(\boldsymbol{\mu}_{c}, \delta \boldsymbol{\mu}\right)=0$ and $I_{2}\left(\boldsymbol{\mu}_{c}, \delta \boldsymbol{\mu}\right)<0$ is the (straight semiline) tangent to the Hopf locus. An iterative predictor corrector scheme, based on the pseudoarclength method, is used again. A point $\boldsymbol{\mu}_{1}$ close to $\boldsymbol{\mu}_{c}$ is taken on one of these two lines (predictor phase); then (corrector phase), the associated invariant is zeroed (i.e., $I_{k}\left(\boldsymbol{\mu}_{1}, \delta \boldsymbol{\mu}\right)=0, k=1$ or 2) together with a linear constraint equation. A new approximation $\boldsymbol{\mu}_{2}=\boldsymbol{\mu}_{1}+\delta \boldsymbol{\mu}$ is obtained, and the procedure reiterated. When convergence has been reached, a new point is predicted on the tangent to follow the curve in the whole region of interest.

By summarizing, the algorithm is as follows:

1) Take the critical point $\boldsymbol{\mu}_{c}$ as initial point $\boldsymbol{\mu}_{0}$ and evaluate the sensitivities $S_{k \mu}\left(\boldsymbol{\mu}_{0}\right)$ and $S_{k v}\left(\boldsymbol{\mu}_{0}\right)(k=1$ or 2$)$ and the vectors $\mathbf{u}_{\mu}^{*}(\boldsymbol{\mu})$ and $\mathbf{u}_{v}^{*}(\boldsymbol{\mu})$ via Eqs. (14) and (15);

2) Select a new point $\mu_{1}$ (predictor phase) on the tangent $\mathbf{t}_{k 0}=$ $\left\{a_{k 0}, b_{k 0}\right\}^{T}$ at $\boldsymbol{\mu}_{0}$, at a sufficiently small distance $|\Delta s|$ from $\boldsymbol{\mu}_{0}$, having coordinates

$$
\mu_{1}=\mu_{0}+a_{k 0} \Delta s, \quad v_{1}=v_{0}+b_{k 0} \Delta s, \quad k=1 \quad \text { or } \quad 2
$$

where

$$
\begin{aligned}
& a_{k 0}:=+\frac{S_{1 v}\left(\boldsymbol{\mu}_{0}\right)}{\sqrt{\left(S_{1 \mu}\right)^{2}\left(\boldsymbol{\mu}_{0}\right)+\left(S_{1 v}\right)^{2}\left(\boldsymbol{\mu}_{0}\right)}} \\
& b_{k 0}:=-\frac{S_{1 \mu}\left(\boldsymbol{\mu}_{0}\right)}{\sqrt{\left(S_{1 \mu}\right)^{2}\left(\boldsymbol{\mu}_{0}\right)+\left(S_{1 v}\right)^{2}\left(\boldsymbol{\mu}_{0}\right)}}, \quad \text { if } k=1
\end{aligned}
$$

or

$$
\begin{gathered}
{\left[\begin{array}{cc}
S_{2 \mu}\left(\boldsymbol{\mu}_{i}\right)-\lambda_{0}\left(\boldsymbol{\mu}_{i}\right) S_{1 \mu}\left(\boldsymbol{\mu}_{i}\right) & S_{2 v}\left(\boldsymbol{\mu}_{i}\right)-\lambda_{0}\left(\boldsymbol{\mu}_{i}\right) S_{1 v}\left(\boldsymbol{\mu}_{i}\right) \\
a_{k 0} & b_{k 0}
\end{array}\right]} \\
\times\left(\begin{array}{c}
\mu_{i+1}-\mu_{i} \\
v_{i+1}-v_{i}
\end{array}\right)=\left(\begin{array}{c}
\lambda_{0}^{2}(\boldsymbol{\mu})-\xi(\boldsymbol{\mu}) \\
\Delta s
\end{array}\right), \quad \text { if } k=2
\end{gathered}
$$

4) If $\left|\boldsymbol{\mu}_{i+1}-\boldsymbol{\mu}_{i}\right|>$ toll $_{1}$ and $\left|\operatorname{Re}\left(\lambda_{k}\left(\boldsymbol{\mu}_{i+1}\right)\right)\right|>\operatorname{toll}_{2}(k=1$ or 2$)$, then execute a new iteration equation (22) or equation (23); if, instead, $\left|\boldsymbol{\mu}_{i+1}-\boldsymbol{\mu}_{i}\right| \leq$ toll $_{1}$ or $\left|\operatorname{Re}\left(\lambda_{k}\left(\boldsymbol{\mu}_{i+1}\right)\right)\right| \leq$ toll $_{2}(k=\overline{1}$ or 2$)$, then assume as a new point $\boldsymbol{\mu}_{0}=\boldsymbol{\mu}_{i+1}$, and restart from step 2 .

\section{Numerical Examples}

Numerical examples are worked out in this section to validate the algorithm via a comparison with known analytical solutions. They concern 1) low dimensional naturally discrete systems and 2) higher dimensional systems obtained by discretization of continuous structures.

Before starting a numerical investigation based on iterative procedures, it is recommended to carry out sensitivity analyses of the results to the tolerances toll $\mathrm{l}_{1,2}$ to be used in the algorithms. Even concerning this task, the perturbation method reveals its power. Indeed, when a nondefective codimension 2 bifurcation point is analyzed, since $\lambda(\boldsymbol{\mu}+\delta \boldsymbol{\mu})-\lambda(\boldsymbol{\mu})=\mathcal{O}(\delta \boldsymbol{\mu})$, a certain accepted error on $\operatorname{Re}[\lambda]=0$ entails an error of the same magnitude on the critical point on $\boldsymbol{\mu}_{c}$. In contrast, when a defective codimension 2 bifurcation point is studied, since $\lambda(\boldsymbol{\mu}+\delta \boldsymbol{\mu})-\lambda(\boldsymbol{\mu})=\mathcal{O}\left(\delta \boldsymbol{\mu}^{1 / 2}\right)$, the same error entails a smaller error on the critical point. Similar arguments hold for the codimension 1 loci construction. All these theoretical considerations were confirmed by preliminary numerical tests. In particular, it was found that, even reducing the tolerances to $10^{-2}$, nearly indistinguishable linear stability diagrams were obtained. Finally, tolerances of $10^{-4}$ were selected.

\section{A. Two-Dimensional Systems}

The double pendulums illustrated in Fig. 2 are considered. The rods are rigid and massless, with masses $m$ lumped at the ends; the elastic springs $k_{i}$ and the dashpots $c_{i}$ are linear. System 1 (in Fig. 2a) is loaded by a deadweight $P$ and a follower force $F$; system 2 (in Fig. 2b) is loaded by a follower force $F$ only. The (nondimen sionalized) follower force $F$ is taken as the $\mu$ parameter, while the (nondimensionalized) dead load $P$, or the stiffness $k_{2}$, are taken as the $v$ parameter for the two systems, respectively.

By choosing the rotations $q_{i}(i=1,2)$ of the two rods as the Lagrangian parameter and $\mathbf{x}=\left(q_{1}, \dot{q}_{1}, q_{2}, \dot{q}_{2}\right)^{T}$ as the state vector, the equations of motion, linearized around the trivial configuration, appear in the form of Eq. (1), where the Jacobian matrix $\mathbf{A}$ is

$$
\begin{aligned}
& a_{k 0}:=+\frac{S_{2 v}\left(\boldsymbol{\mu}_{0}\right)-\lambda_{0}\left(\boldsymbol{\mu}_{0}\right) S_{1 v}\left(\boldsymbol{\mu}_{0}\right)}{\sqrt{\left(S_{2 \mu}\left(\boldsymbol{\mu}_{0}\right)-\lambda_{0}\left(\boldsymbol{\mu}_{0}\right) S_{1 \mu}\left(\boldsymbol{\mu}_{0}\right)\right)^{2}+\left(S_{2 v}\left(\boldsymbol{\mu}_{0}\right)-\lambda_{0}\left(\boldsymbol{\mu}_{0}\right) S_{1 v}\left(\boldsymbol{\mu}_{0}\right)\right)^{2}}}, \\
& b_{k 0}:=-\frac{S_{2 \mu}\left(\boldsymbol{\mu}_{0}\right)-\lambda_{0}\left(\boldsymbol{\mu}_{0}\right) S_{1 \mu}\left(\boldsymbol{\mu}_{0}\right)}{\sqrt{\left(S_{2 \mu}\left(\boldsymbol{\mu}_{0}\right)-\lambda_{0}\left(\boldsymbol{\mu}_{0}\right) S_{1 \mu}\left(\boldsymbol{\mu}_{0}\right)\right)^{2}+\left(S_{2 v}\left(\boldsymbol{\mu}_{0}\right)-\lambda_{0}\left(\boldsymbol{\mu}_{0}\right) S_{1 v}\left(\boldsymbol{\mu}_{0}\right)\right)^{2}}}, \quad \text { if } k=2
\end{aligned}
$$

3) Solve iteratively (for $i=1,2, \ldots$ ) the following equations in the unknowns $\mu_{i+1}$ and $v_{i+1}$ (corrector phase):

$$
\left[\begin{array}{cc}
S_{1 \mu}\left(\boldsymbol{\mu}_{i}\right) & S_{1 v}\left(\boldsymbol{\mu}_{i}\right) \\
a_{k 0} & b_{k 0}
\end{array}\right]\left(\begin{array}{c}
\mu_{i+1}-\mu_{i} \\
v_{i+1}-v_{i}
\end{array}\right)=\left(\begin{array}{c}
-2 \lambda_{0}\left(\boldsymbol{\mu}_{i}\right) \\
\Delta s
\end{array}\right)
$$

if $k=1$

$$
\mathbf{A}=\left[\begin{array}{cccc}
0 & 1 & 0 & 0 \\
-3 \kappa+\mu+v & -3 \eta & 2 \kappa-\mu-v & 2 \eta \\
0 & 0 & 0 & 1 \\
4 \kappa-\mu-v & 4 \eta & -3 \kappa+\mu+2 v & -3 \eta
\end{array}\right]
$$




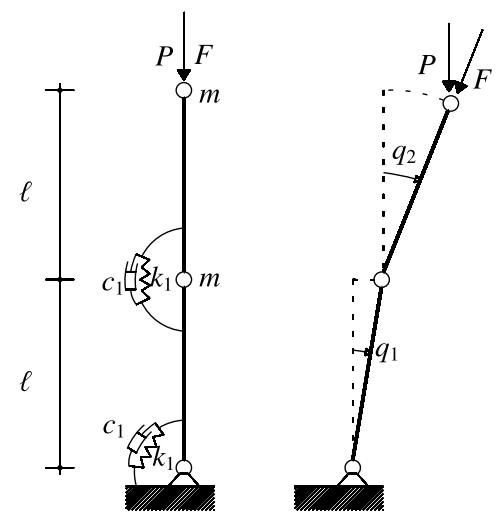

a)

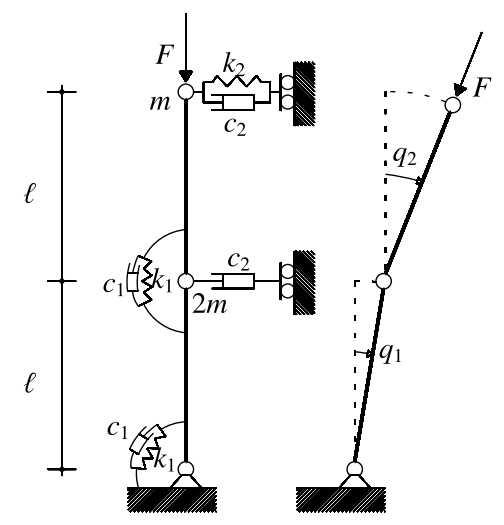

b)

Fig. 2 Double pendulum a) loaded by a follower force and a dead load and b) loaded by a follower force and viscoelastically braced.

$$
\mathbf{A}=\left[\begin{array}{cccc}
0 & 1 & 0 & 0 \\
\frac{-3 \kappa+\mu}{2} & \frac{\eta(-3-\zeta)}{2} & \frac{2 \kappa-\mu}{2} & \eta \\
0 & 0 & 0 & 1 \\
\frac{\kappa \kappa-2 v-\mu}{2} & \frac{\eta(5-\zeta)}{2} & \frac{\mu-4 \kappa-2 v}{2} & \eta(-2-\zeta)
\end{array}\right]
$$

for system 2. In Eqs. (24) and (25), the following nondimensional parameters appear: $\kappa:=k_{1} /\left(m \omega^{2} \ell^{2}\right), \eta:=c_{1} /\left(m \omega \ell^{2}\right)$, and $\mu:=$ $F /\left(m \omega^{2} \ell\right)$ for both systems; $v:=P /\left(m \omega^{2} \ell\right)$ for system 1 ; and $\zeta=c_{2} \ell^{2} / c_{1}$ and $v:=k_{2} /\left(m \omega^{2}\right)$ for system 2 . Selected numerical values are taken for the auxiliary parameters, namely, $\eta=0.1$ and $\kappa=1$ for system 1 ; and $\eta=1.5, \kappa=1$, and $\zeta=0.5$ for system 2 .

From the characteristic polynomial of the Jacobian matrix in Eq. (24), it follows that system 1 undergoes a divergence Hopf bifurcation at $\boldsymbol{\mu}_{c}=(-2.35,5.77)$, i.e., for tensile dead load and compressive follower force. By applying the procedure of Sec. II.B, the iterations displayed in Fig. 3a were performed, starting from points $\boldsymbol{\mu}_{0}$ lying on circles centered at $\boldsymbol{\mu}_{c}$ having radii $\left|\boldsymbol{\mu}_{0}-\boldsymbol{\mu}_{c}\right|=$ $0.3,0.5$, respectively. Convergence was reached in a few steps from all points of the smallest circle (unfilled symbols in the figure), while some divergent iterations were observed from the largest circle (filled symbols). Then, starting from $\boldsymbol{\mu}_{c}$, and applying the procedure of Sec. II.C, the bifurcation loci depicted in Fig. 3 b were obtained. Here, the region of Fig. 3a is also reported.

From the Jacobian matrix of Eq. (25), it is found that system 2 experiences a double zero (DZ) bifurcation at $\mu_{c}=(0.15,5.83)$. A tentative point $\boldsymbol{\mu}_{0}=(0.08,8)$ was chosen, and both the iterative procedure for nondefective and defective systems were applied. As it appears in Fig. 4a, the algorithm based on sensitivity of the distinct eigenvalues diverges, while the method grounded on nearly coincident eigenvalue converges fast. The bifurcation loci origi nating from $\boldsymbol{\mu}_{c}$ are shown in Fig. $4 \mathrm{~b}$.

The exact bifurcation loci can also be drawn by the characteristic polynomials of the Jacobian matrices [Eqs. (24) and (25)] by

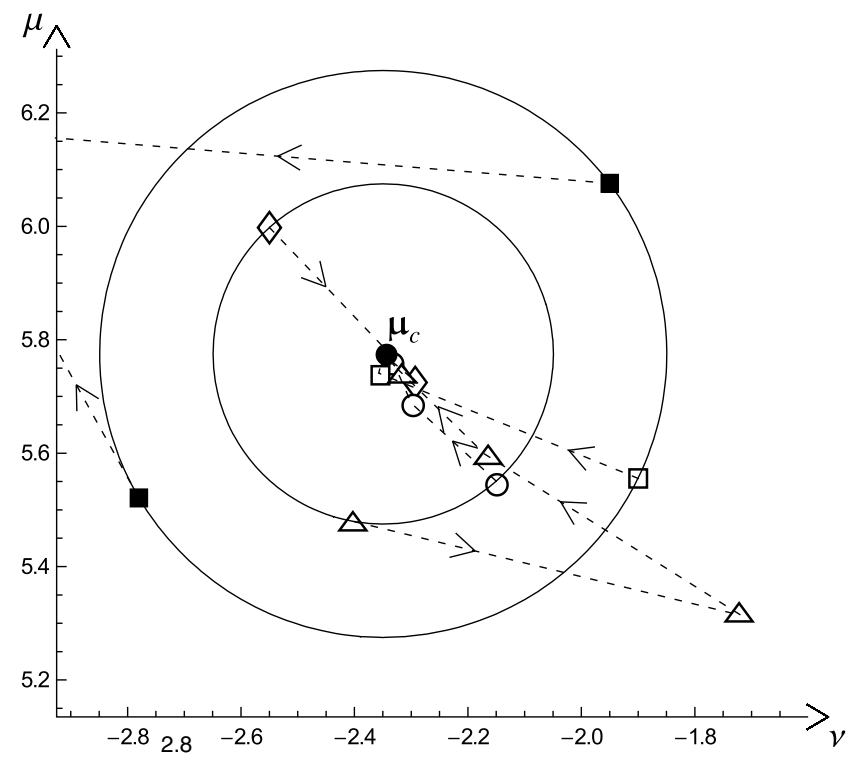

a)

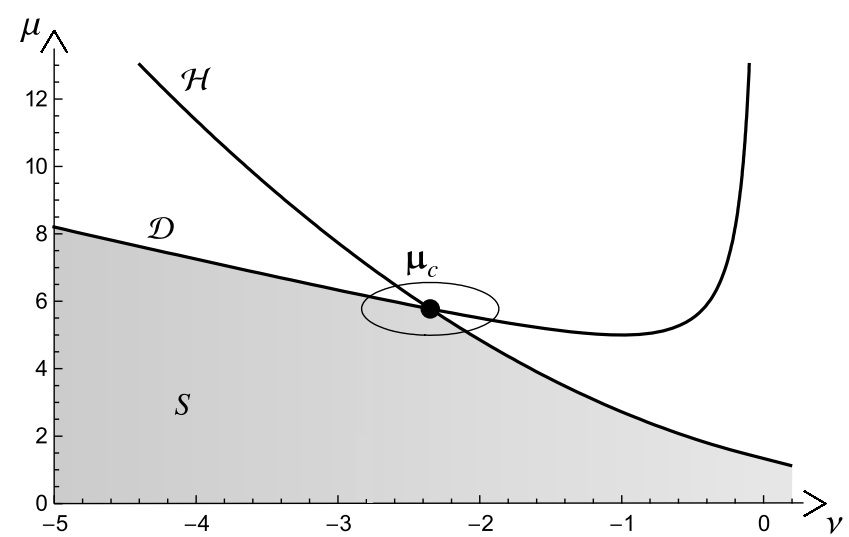

b)

Fig. 3 System 1: a) iterations to divergence-Hopf bifurcation point and b) bifurcation loci (; $S$ : stable region).

applying the Routh Hurwitz criterion. For system 1, they turn out to have the following equations (where $\mathcal{D}$ is divergence locus and $\mathcal{H}$ is Hopf locus):

$$
\begin{aligned}
& \mathcal{D}: v=\frac{1}{2}\left(3-\mu \pm \sqrt{5-6 \mu-\mu^{2}}\right) \\
& \mathcal{H}: v=0.33(4.03 \pm \sqrt{-1.76+2.67 \mu})
\end{aligned}
$$

and, for system 2 ,

$$
\begin{aligned}
& \mathcal{D}: \mu=\frac{1+5 v}{v} \\
& \mathcal{H}: \mu=6.74-1.41 v-1.41 \sqrt{(0.16-v)(23.78+v)}
\end{aligned}
$$

Boundaries in Fig. $3 \mathrm{~b}$ and in Fig. $4 \mathrm{~b}$ are indistinguishable (in this scale) from the exact bifurcation loci equations (26) and (27).

\section{B. Discretized Continuous Systems}

To test the algorithm on larger systems, two different continuous beams are studied, for which analytical solutions are available, and a Galerkin spatial discretization is then performed.

The structure depicted in Fig. 5a (system 3 ) consists of a planar viscoelastic beam, fixed at end $\bar{A}$, constrained by a linear elastic spring of stiffness $K$ at end $B$, and loaded at the tip by a follower force of intensity $F$. The material behavior is assumed to be described by the Kelvin Voigt rheological model, having elastic modulus $E$ and 


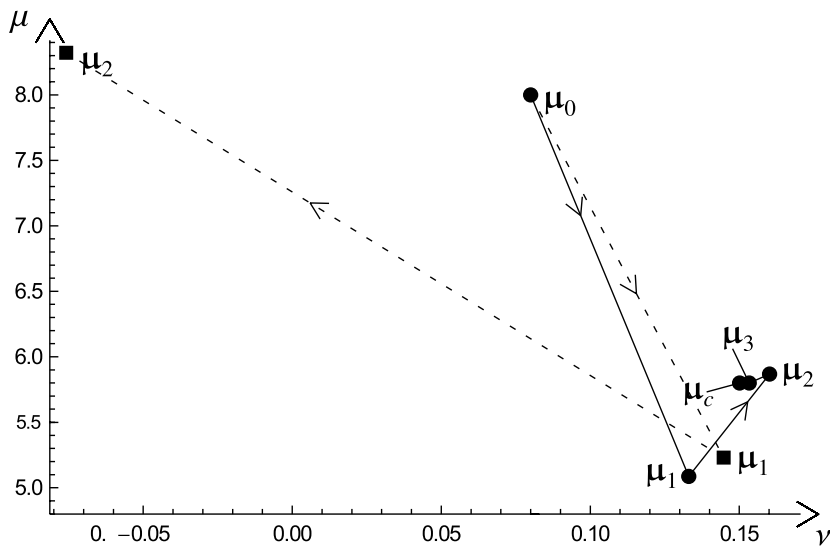

a)

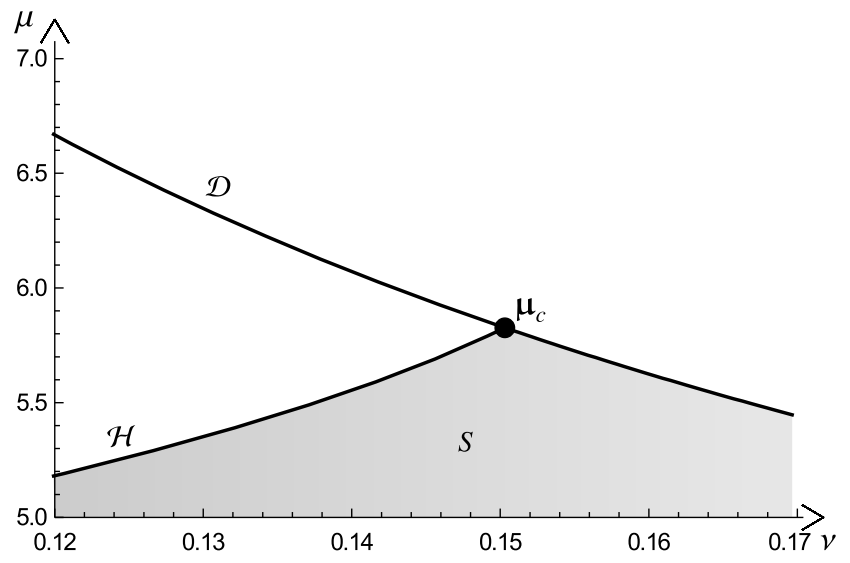

b)

Fig. 4 System 2: a) iterations to the DZ bifurcation point (O: sensitivity for nearly coincident eigenvalues; $\square$ : sensitivity for distinct eigenvalues) and b) bifurcation loci ( $S$ : stable region).

viscous coefficient $\eta$ (acting as an internal damping); moreover, the beam is considered to lie on a linear purely viscous soil of constant $c$ (representing the external damping). If damping were not present at all, the system would coincide with that studied in [10], where a degenerate (not generic) tangential bifurcation occurs, which is not analyzable by the theory developed here.

A similar structure is considered in Fig. 5b (system 4), obtained by the previous one by removing all distributed damping and lumping it at the tip of the beam by means of two linear dashpots of constants $C_{e}$ and $C_{t}$ of extensional and torsional types, respectively. System 4 was chosen to test the algorithm in a difficult case, since it was shown by analytical methods in [11] that this beam exhibits a rich bifurcation scenario containing several bifurcation points of different natures close to each other.

Both beams are assumed to be inextensible and shear undeform able, so that their deformed configurations are described by the transversal displacement field $u(s, t)$ only. A Galerkin discretization is performed by assuming

$$
u(s, t)=\sum_{k=1}^{n} q_{k}(t) \phi_{k}(s)
$$

with $\phi_{k}(s)$ known shape functions satisfying kinematic boundary conditions and $q_{k}(t)$ unknown amplitudes leading to linearized equations of motion, as in Eq. (1) (see Appendix B for formulation of the variational principle). In them, $\mathbf{x}=\left(q_{1}, q_{2}, \ldots, q_{n} ; \dot{q}_{1}, \dot{q}_{2}\right.$, $\left.\ldots, \dot{q}_{n}\right)^{T}$ is the $2 n$ vector of the Lagrangian coordinates, $\mathbf{A}=$ $\mathbf{A}(\mu, v)$ is the $(2 n \times 2 n)$ Jacobian matrix, and $\mu$ and $v$ are the bifurcation parameters representing, for both systems, the non dimensional tangential force $F$ and the nondimensional spring stiffness $K$, respectively.

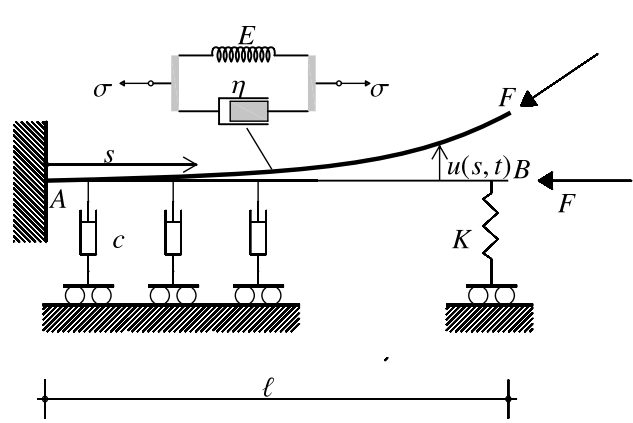

a)

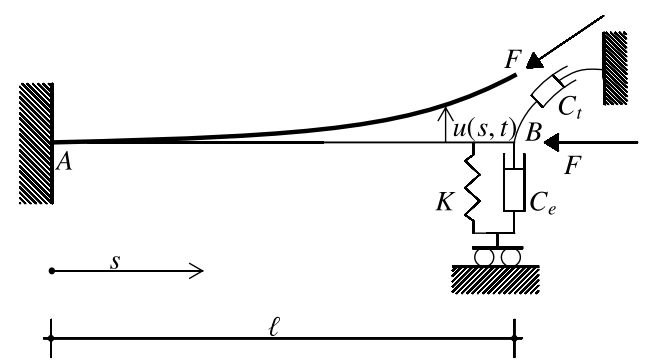

b)

Fig. 5 Planar beam under follower force: a) viscoelastic beam on viscous soil, elastically braced, and b) elastic beam viscoelastically braced.

\section{Viscoelastic Beam}

System 3 (Fig. $\underline{5 \text { a) }}$ is now analyzed. The first question to address concerns the choice of the guess point $\mu_{0}$ from which to start the iterations. Although a hint about $\mu_{0}$ could be derived by an analytical solution for the original continuous system, a different strategy is followed here in view of problems in which no exact solutions are available, namely:

1) A rough discretization is preliminarily performed, leading to a low dimensional system.

2) The Routh Hurwitz criterion is applied to gain coarse information about the location of the codimension 2 bifurcation point (if any).

3) The discretization is refined, and the iteration is started by the point previously determined.

A rough discretization with $n=2$ shape functions (four dimensional system) was performed. The first two buckling modes of the (unbraced) cantilever under a conservative (dead load) axial force were tentatively used. However, this coarse discretization lead to an illusory Hopf divergence bifurcation point, which was not confirmed by higher order discrete systems. Therefore, more accurate shape functions were selected as the two first buckling modes of the elastically braced cantilever under nonconservative (follower) load. For a chosen value $(v=40)$ of the spring, they come out to be

$$
\begin{aligned}
& \phi_{1}(s)=1+3.36 s-\cos (5.27 s)-0.64 \sin (5.28 s) \\
& \phi_{2}(s)=1-235.46 s-\cos (6.31 s)+37.31 \sin (6.31 s)
\end{aligned}
$$

From the Routh Hurwitz criterion, it follows that the four dimensional system admits a divergence and a Hopf loci having equations

$$
\begin{aligned}
& \mathcal{D}: \mu=0.18 v \pm 0.18 \sqrt{(v-4.81)(v-31.98)}+9.81 \\
& \mathcal{H}: \mu=0.28 v+0.55 \sqrt{v^{2}-281.84 v+2.2 \cdot 10^{6}}-808.84
\end{aligned}
$$

intersecting each other at a DZ bifurcation point of coordinates DZ: $\boldsymbol{\mu}_{c}=(34.61,17.54)$

The two loci in Eq. (29) are plotted in Fig. 6. If the proposed algorithm is started from a point close to DZ, the same curves are recovered for the four dimensional system. 


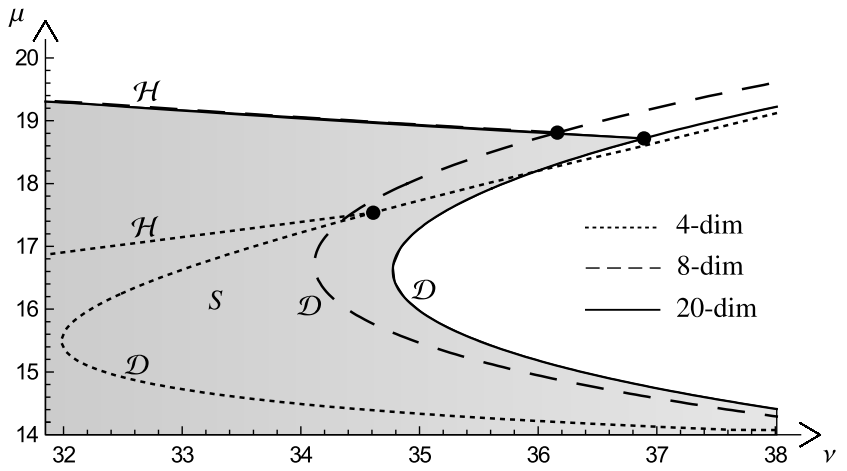

Fig. 6 System 3: bifurcation point and loci for different discretizations $(\mathcal{D}$ : divergence locus; $\mathcal{H}$ : Hopf locus; $S$ : stable region; $\mu$ : nondimensional follower force parameter; $v$ : nondimensional stiffness parameter; dim: dimensional).

To refine the discretization, however, buckling modes of the braced beam under follower force cannot be used, since they do not constitute a complete system (their number is limited when $v$ is different from infinite, as it can be easily checked by the characteristic equation). Therefore, use was made again of the buckling modes of the unbraced beam under dead load:

$$
\phi_{k}(s)=1-\cos \left[(2 k-1) \frac{\pi s}{2}\right], \quad k=1,2, \ldots, n
$$

and convergence was checked for increasing $n$. Results furnished by the implemented numerical algorithm are displayed in Fig. 6 and compared with that of the coarse system for eight dimensional $(n=4)$ and 20 dimensional $(n=10)$ systems. Curves were obtained by adopting the starting point $\boldsymbol{\mu}_{0}=(34.61,17.54)$, thus finding improved approximations for the DZ point, namely, DZ: $\boldsymbol{\mu}_{c}=$ $(36.16,18.81)$ for the eight dimensional system and DZ: $\mu_{c}=$ $(36.89,18.72)$ for the 20 dimensional one. When these results are compared with the analytical solution (see Appendix C), the more refined solution is nearly undistinguishable from the exact one.

\section{Elastic Beam with Lumped Damping}

System 4 (Fig. 5b) is now addressed (see Appendix B for formulation). For this beam, the presence of the viscous device at the tip calls for selecting shape functions $\phi_{k}(s)$ able to properly account for the boundary conditions at $B$. Indeed, it was found that an inaccurate choice of them would lead to erroneous results. Here, consistently with the procedure suggested in [12], the following shape functions were selected:

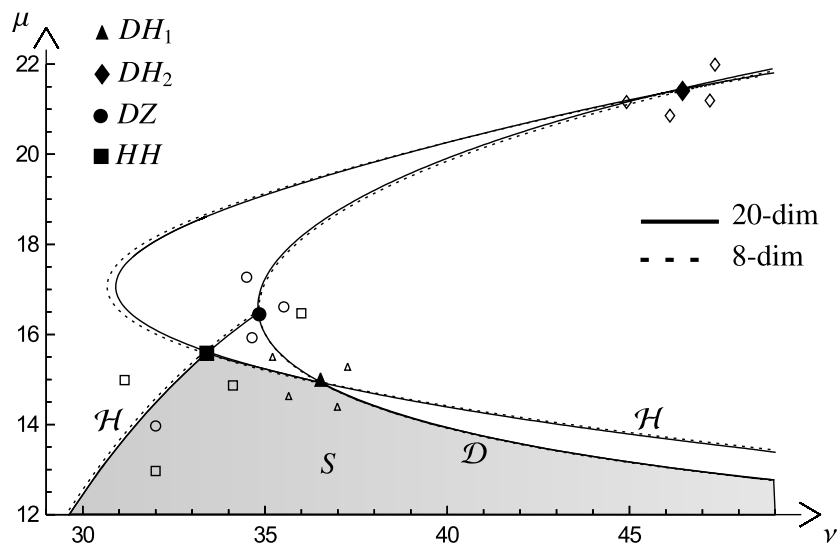

Fig. 7 System 4: iterations to bifurcation points and bifurcation loci (D) divergence locus; $\mathcal{H}$ : Hopf locus; $S$ : stable region; $\mu$ : nondimensional follower force parameter; $v$ : nondimensional stiffness parameter; dim: dimensional).
Table 1 Relative error $\varepsilon \%$ of the critical points vs discretization degree

\begin{tabular}{lccc}
\hline \hline & $\boldsymbol{\Delta} \mathrm{DH}_{1}$ & $\boldsymbol{\bullet} \mathrm{DZ}$ & $\boldsymbol{\square} \mathrm{HH}$ \\
\hline 8 dimensional & 2.87 & 1.25 & 2.53 \\
20 dimensional & 0.31 & 0.15 & 0.77 \\
\hline
\end{tabular}

$$
\begin{gathered}
\phi_{k}(s)=1-\cos \left[(2 k-1) \frac{\pi s}{2}\right], \quad k=1,2, \ldots, n-1 \\
\phi_{n}(s)=s^{2}-\sum_{k=1}^{n-1} c_{k} \phi_{k}(s)
\end{gathered}
$$

where

$$
c_{k}=\left[\int_{o}^{1} s^{2} \phi_{k}(s) \mathrm{d} s\right] /\left[\int_{o}^{1} \phi_{k}^{2}(s) \mathrm{d} s\right]
$$

are the coefficients of the series expansion of $s^{2}$ in the basis $\left\{\phi_{k}(s)\right\}$. The first $n-1$ elements of this set are the buckling eigenfunctions of a cantilevered beam under a conservative axial load; the $n$th element is a residue localized at the tip, obtained as the difference between a parabola and its projection on the space spanned by the first $n-1$ elements. The motivations for appending Eqs. (31a) and (31b) are discussed in Appendix D.

The scenario furnished by the algorithm is displayed in Fig. 7 for two different discretizations, namely, the eight dimensional system $(n=4)$ and the 20 dimensional system $(n=10)$. Different kinds of bifurcations, both defective and nondefective, are found to occur in the parameter space. In particular, the algorithm finds four bifurcation points (marked with filled symbols in Fig. 7), namely, two divergence Hopf points $\left(\mathrm{DH}_{1,2}\right)$, a DZ, and a Hopf $\overline{H o p f}(\mathrm{HH})$ point. For the eight dimensional system, the algorithm furnishes the following bifurcation points: $\mathrm{DH}_{1}: \mu_{c}=(36.43,14.96), \mathrm{DH}_{2}: \mu_{c}=$ $(46.86,21.48), \mathrm{DZ}: \boldsymbol{\mu}_{c}=(34.83,16.53)$, and $\mathrm{HH}: \boldsymbol{\mu}_{c}=(33.36$, 15.62). For the 20 dimensional system, they are $\mathrm{DH}_{1}: \mu_{c}=$ (36.55, 14.92), $\quad \mathrm{DH}_{2}: \boldsymbol{\mu}_{c}=(45.88,21.35), \quad \mathrm{DZ}: \boldsymbol{\mu}_{c}=(34.81$, $16.47)$, and $\mathrm{HH}: \boldsymbol{\mu}_{c}=(33.44,15.61)$. Some of the starting points used in the algorithm are also shown in Fig. 7, represented by the same unfilled symbol of the target point. In all cases, the procedure converges after few iterations if the starting point is sufficiently close to the bifurcation point. These results are compared with the exact solutions furnished in [11] in order to check the error, both due to the discretization procedure and to the tolerances of the algorithm. For both the discretizations considered, the comparison is excellent, as shown in Table 1.

The bifurcation loci originating from the various critical points are shown in Fig. 7 for the two discretizations adopted. They are very close to each other; moreover, the more refined solution is nearly undistinguishable from the exact solution given in [11].

\section{Conclusions}

By exploiting the potentiality of an eigenvalue sensitivity analysis, an iterative numerical perturbation method was implemented to build up linear stability diagrams of two parameter dynamical systems undergoing codimension 2 bifurcations. Both nondefective and defective bifurcations were studied, and specific algorithms were illustrated. Numerical examples were presented, relevant to discrete or discretized mechanical systems, exhibiting Hopf divergence, Hopf Hopf, or DZ bifurcations. The following conclusions were drawn:

1) The new method consists of two steps:

a) Find the codimension 2 bifurcation point.

b) Build up the branches emanating from this point.

2) The method reverses the usual approach, according to which one first looks for codimenson 1 bifurcation loci and then follows them by checking, at each step, if a codimension 2 point is encountered. 
3) Although, in the proposed method, the initial point of a Newton procedure is likely more difficult to be determined, it was found that the convergence to the codimension 2 bifurcation point is generally fast when a sufficiently close initial guess of the point is chosen.

4) A strategy was suggested to facilitate the choice of the starting point. It consists of a preliminary coarse (few degrees of freedom) modeling of the system, making the search of the critical point feasible via a Routh Hurwitz analysis. A successive numerical analysis, carried out on a refined model, improves this result.

5) The algorithm gives results in excellent agreement with known analytical solutions, when available.

6) Some interesting problems related to the choice of the shape functions adopted in the discretizing continuous system were discussed.

\section{Appendix A: Eigenvalue Sensitivity Analysis}

Sensitivity analysis consists of finding closed form expressions for the eigenpairs $(\tilde{\lambda}, \tilde{\mathbf{x}})$ of a parameter dependent (perturbed) matrix $\tilde{\mathbf{A}}=\mathbf{A}(\boldsymbol{\mu}+\varepsilon \delta \boldsymbol{\mu}) \quad$ (with $\varepsilon \ll 1$ and $|\delta \boldsymbol{\mu}|=\mathcal{O}(1)$ ) once the eigenpairs $\left(\lambda_{0}, \mathbf{x}_{0}\right)$ of a (unperturbed) matrix $\mathbf{A}_{0}:=\mathbf{A}(\boldsymbol{\mu})$ are known. Accordingly, the solutions of the eigenvalue problem $(\tilde{\mathbf{A}}-$ $\tilde{\lambda} \mathbf{I}) \tilde{\mathbf{x}}=\mathbf{0}$ are sought as a perturbation of the solutions of $\left(\mathbf{A}_{0}-\lambda_{0} \mathbf{I}\right) \mathbf{x}_{0}=\mathbf{0}$. Here, two different cases are shortly analyzed:

1) Variable $\lambda_{0}$ is a simple eigenvalue.

2) Variable $\lambda_{0}$ is a double defective eigenvalue; that is, only one eigenvector is associated with it.

Then, the case of two nearly coincident eigenvalues with nearly coincident eigenvectors is addressed.

\section{Sensitivity of a Simple Eigenvalue}

Matrix $\tilde{\mathbf{A}}$ and its eigenpairs are expanded in a series of perturbation parameter $\varepsilon$ :

$$
\begin{gathered}
\tilde{\mathbf{A}}=\mathbf{A}_{0}+\varepsilon \mathbf{A}_{1}+\mathcal{O}\left(\varepsilon^{2}\right) \quad \tilde{\lambda}=\lambda_{0}+\varepsilon \lambda_{1}+\mathcal{O}\left(\varepsilon^{2}\right) \\
\tilde{\mathbf{x}}=\mathbf{x}_{0}+\varepsilon \mathbf{x}_{1}+\mathcal{O}\left(\varepsilon^{2}\right)
\end{gathered}
$$

where $\mathbf{A}_{1}=\mathbf{A},{ }_{\mu}(\boldsymbol{\mu}) \delta \boldsymbol{\mu}$. Substitution in the eigenvalue problem and collection of the same order terms leads to the following perturbation equations:

$$
\begin{gathered}
\varepsilon^{0}:\left(\mathbf{A}_{0}-\lambda_{0} \mathbf{I}\right) \mathbf{x}_{0}=\mathbf{0} \\
\varepsilon:\left(\mathbf{A}_{0}-\lambda_{0} \mathbf{I}\right) \mathbf{x}_{1}=\lambda_{1} \mathbf{x}_{0}-\mathbf{A}_{1} \mathbf{x}_{0}
\end{gathered}
$$

Equation (A2a) supplies the unperturbed $\lambda_{0}$ and $\mathbf{x}_{0}$. Then, solvability of Eq. (A $\overline{2 b}$ ) requires the known term to be orthogonal to the left eigenvector $\mathbf{y}_{0}$ [solution to $\left(\mathbf{A}_{0}-\lambda_{0} \mathbf{I}\right)^{H} \mathbf{y}_{0}=\mathbf{0}$ ], from which the first sensitivity follows:

$$
\lambda_{1}=\mathbf{y}_{k 0}^{H} \mathbf{A}_{1} \mathbf{x}_{k 0}
$$

In Eq. (A3), the normalization condition $\mathbf{y}_{k 0}^{H} \mathbf{x}_{k 0}=1$ has been used. Equation (3) is grounded in Eq. (A3).

\section{Sensitivity of a Defective Double Eigenvalue}

In this case, generalized right and left eigenvectors exist for $\mathbf{A}_{0}$, solving the recurrence equations:

$$
\begin{gathered}
\left(\mathbf{A}_{0}-\lambda_{0} \mathbf{I}\right) \mathbf{x}_{10}=\mathbf{0} \\
\left(\mathbf{A}_{0}-\lambda_{0} \mathbf{I}\right) \mathbf{x}_{20}=\mathbf{x}_{10} \\
\left(\mathbf{A}_{0}-\lambda_{0} \mathbf{I}\right)^{H} \mathbf{y}_{20}=\mathbf{0} \\
\left(\mathbf{A}_{0}-\lambda_{0} \mathbf{I}\right)^{H} \mathbf{y}_{10}=\mathbf{y}_{20}
\end{gathered}
$$

Here, $\mathbf{x}_{10}$ and $\mathbf{y}_{20}$ are proper eigenvectors, and $\mathbf{x}_{20}$ and $\mathbf{y}_{10}$ are index 2 eigenvectors, satisfying the orthonormalization conditions $\mathbf{y}_{i 0}^{H} \mathbf{x}_{j 0}=\delta_{i j}$. The following series expansions hold for $\tilde{\mathbf{A}}$ and its eigenpairs:

$$
\begin{gathered}
\tilde{\mathbf{A}}=\mathbf{A}_{0}+\varepsilon \mathbf{A}_{1}+\mathcal{O}\left(\varepsilon^{2}\right) \\
\tilde{\lambda}=\lambda_{0}+\varepsilon^{1 / 2} \lambda_{1}+\varepsilon \lambda_{2}+\varepsilon^{3 / 2} \lambda_{3}+\mathcal{O}\left(\varepsilon^{2}\right) \\
\tilde{\mathbf{x}}=\mathbf{x}_{0}+\varepsilon^{1 / 2} \mathbf{x}_{1}+\varepsilon \mathbf{x}_{2}+\varepsilon^{3 / 2} \mathbf{x}_{3}+\mathcal{O}\left(\varepsilon^{2}\right)
\end{gathered}
$$

With Eqs. (A6), the eigenvalue problem furnishes the following perturbation equations:

$$
\begin{gathered}
\varepsilon^{0}:\left(\mathbf{A}_{0}-\lambda_{0} \mathbf{I}\right) \mathbf{x}_{0}=\mathbf{0} \\
\varepsilon^{1 / 2}:\left(\mathbf{A}_{0}-\lambda_{0} \mathbf{I}\right) \mathbf{x}_{1}=\lambda_{1} \mathbf{x}_{0} \\
\varepsilon:\left(\mathbf{A}_{0}-\lambda_{0} \mathbf{I}\right) \mathbf{x}_{2}=\lambda_{1} \mathbf{x}_{1}+\lambda_{2} \mathbf{x}_{0}-\mathbf{A}_{1} \mathbf{x}_{0} \\
\varepsilon^{3 / 2}:\left(\mathbf{A}_{0}-\lambda_{0} \mathbf{I}\right) \mathbf{x}_{3}=\lambda_{1} \mathbf{x}_{2}+\lambda_{2} \mathbf{x}_{1}+\lambda_{3} \mathbf{x}_{0}-\mathbf{A}_{1} \mathbf{x}_{1}
\end{gathered}
$$

By solving them in a chain, using Eq. (A4b), and enforcing solvability at the $\varepsilon$ and $\varepsilon^{3 / 2}$ orders, the following conditions are obtained:

$$
\lambda_{1}^{2}=\mathbf{y}_{20}^{H} \mathbf{A}_{1} \mathbf{x}_{10} \quad 2 \lambda_{1} \lambda_{2}=\lambda_{1} \mathbf{y}_{20}^{H}\left(\mathbf{A}_{1} \mathbf{x}_{20}-\mathbf{u}^{*}\right)
$$

in which $\mathbf{u}^{*}$ is a particular solution of Eq. (A7c). They could be solved for $\lambda_{1}$ and $\lambda_{2}$ separately, but this solution would break down when $\lambda_{1}$ is zero or small (see [13] for further comments). Therefore, it is more convenient to recombine them (reconstitution principle) in a second degree sensitivity equation in the increment $\Delta \lambda: \tilde{\lambda}-\lambda_{0}$. By observing that $\Delta \lambda^{2}=\varepsilon \lambda_{1}^{2}+2 \varepsilon^{3 / 2} \lambda_{1} \lambda_{2}+\mathcal{O}\left(\varepsilon^{2}\right)$ and $\Delta \lambda: \varepsilon^{1 / 2} \lambda_{1}+$ $\mathcal{O}(\varepsilon)$, retaining terms up to $\mathcal{O}\left(\varepsilon^{3 / 2}\right)$, and reabsorbing $\varepsilon$, the following sensitivity equation is obtained:

$$
\Delta \lambda^{2}-\mathbf{y}_{20}^{H}\left(\mathbf{A}_{1} \mathbf{x}_{20}-\mathbf{u}^{*}\right) \Delta \lambda-\mathbf{y}_{20}^{H} \mathbf{A}_{1} \mathbf{x}_{10}=0
$$

\section{Sensitivity of Nearly Coincident Eigenvalues}

Let us consider a nearly defective matrix $\hat{\mathbf{A}}:=\mathbf{A}(\hat{\boldsymbol{\mu}})$ admitting nearly coincident eigenvalues $\hat{\lambda}_{1}, \hat{\lambda}_{2}$ associated with nearly coincident eigenvectors $\hat{\mathbf{x}}_{1}, \hat{\mathbf{x}}_{2}$ and $\hat{\mathbf{y}}_{1}, \hat{\mathbf{y}}_{2}$. This case can be studied (see [9]) by first looking for an (exactly) defective matrix $\mathbf{A}_{0}:=\hat{\mathbf{A}}-\xi \mathbf{A}_{\xi}$, in which $\xi \in \mathbb{R}$ is a small mistuning parameter and $\mathbf{A}_{\xi}$ a properly chosen matrix (inverse problem); after that, the sensitivities of $\lambda_{0}$ must be evaluated as a function of both $\delta \boldsymbol{\mu}$ and $\xi$. According to [9], it is found that

$$
\xi=\frac{1}{4}\left(\hat{\lambda}_{1}-\hat{\lambda}_{2}\right)^{2} \quad \mathbf{A}_{\xi}:=\mathbf{x}_{20}(\boldsymbol{\mu}) \mathbf{y}_{10}^{H}(\boldsymbol{\mu})
$$

where

$$
\mathbf{x}_{20}=\frac{1}{2} \xi^{-1 / 2}\left(\hat{\mathbf{x}}_{1}-\hat{\mathbf{x}}_{2}\right) \quad \mathbf{y}_{10}=\hat{\mathbf{y}}_{1}+\hat{\mathbf{y}}_{2}
$$

From Eqs. (6a) and (A11), the perturbed matrix then reads

$$
\tilde{\mathbf{A}}=\hat{\mathbf{A}}+\varepsilon \mathbf{A},{ }_{\mu} \delta \boldsymbol{\mu}+\mathcal{O}\left(\varepsilon^{2}\right)=\mathbf{A}_{0}+\varepsilon\left(\xi \mathbf{A}_{\xi}+\mathbf{A},{ }_{\mu} \delta \boldsymbol{\mu}\right)+\mathcal{O}\left(\varepsilon^{2}\right)
$$

where the rescaling $\xi \rightarrow \varepsilon \xi$ has been performed. By exploiting results of the previous subsection, the sensitivity equation (A9) still holds, with $\mathbf{A}_{1}$ now redefined as $\mathbf{A}_{1}:=\xi \mathbf{A}_{\xi}+\mathbf{A},{ }_{\mu} \overline{\delta \mu}$, i.e., including the mistuning parameter $\xi$. This form is the one used in Eq. (13). 


\section{Appendix B: Discrete Formulation of Cantilevered Beams}

Systems in Fig. 5a (system 3) and Fig. 5b (system 4) are considered, for which the first variation of extended Hamilton's principle reads

$$
\begin{gathered}
\delta H=\int_{t_{1}}^{t_{2}}\left\{\int_{0}^{1}\left(\dot{u} \delta \dot{u}-u^{\prime \prime} \delta u^{\prime \prime}+2 \mu u^{\prime} \delta u^{\prime}\right) \mathrm{d} s\right. \\
\left.-\left(v u_{B}+2 \mu u_{B}^{\prime}\right) \delta u_{B}+\delta W_{d}\right\} \mathrm{d} t=0
\end{gathered}
$$

where $\delta W_{d}$ is the virtual work done by damping forces; for the two systems, it reads

$$
\begin{aligned}
\delta W_{d} & =\int_{0}^{1}-\left(\alpha \dot{u}^{\prime \prime} \delta u^{\prime \prime}+\beta \dot{u} \delta u\right) \mathrm{d} s \\
\delta W_{d} & =-\xi_{e} \dot{u}_{B} \delta u_{B}-\xi_{t} \dot{u}_{B}^{\prime} \delta u_{B}^{\prime}
\end{aligned}
$$

where $u(s, t)$ is the transversal displacement of the beam at the nondimensional abscissa $s \in[0,1]$ and nondimensional time $t ; \mu:=$ $F \ell^{2} / 2 E I$ and $v:=K \ell^{3} / E I$ (for both systems); $\alpha:=\eta \omega / E$ and $\beta:=c \omega \ell^{4} / E I$ (for system 3); and $\xi_{e}:=C_{e} \omega \ell^{3} / E I$ and $\xi_{t}:=$ $C_{t} \omega \ell / E I$ (for system 4) are nondimensional parameters; $\ell$ is the length of the beam, $E I$ is the bending stiffness, and $\omega=\left(E I / m \ell^{4}\right)^{1 / 2}$ is a frequency. The values of the auxiliary parameters are taken equal to $\alpha=0.4$ and $\beta=0.3$ for system 3 and $\xi_{e}=0.25$ and $\xi_{t}=0.5$ for system 4 .

By using

$$
\begin{gathered}
u(s, t)=\sum_{k=1}^{n} q_{k}(t) \phi_{k}(s) \\
\delta u(s, t)=\sum_{k=1}^{n} \delta q_{k}(t) \phi_{k}(s)
\end{gathered}
$$

into Eq. (B1), and performing standard steps, the equations of motion are found in the form of Eq. (1), where

$$
\mathbf{A}(\mu, v):=\left[\begin{array}{cc}
\mathbf{0} & \mathbf{I} \\
-\mathbf{M}^{-1} \mathbf{K} & -\mathbf{M}^{-1} \mathbf{C}
\end{array}\right]
$$

is the $(2 n \times 2 n)$ Jacobian matrix, $\mathbf{0}$ is the $(n \times n)$ null matrix, $\mathbf{I}$ is the $(n \times n)$ identity matrix, and

$$
\mathbf{M}:=\left[\int_{0}^{1} \phi_{j} \phi_{k} \mathrm{~d} s\right]
$$

$$
\begin{aligned}
\mathbf{K} & :=\left[\int_{0}^{1} \phi_{j}^{\prime \prime}(s) \phi_{k}^{\prime \prime}(s) \mathrm{d} s-2 \mu \int_{0}^{1} \phi_{j}^{\prime}(s) \phi_{k}^{\prime}(s) \mathrm{d} s+v \phi_{j}(1) \phi_{k}(1)\right. \\
& \left.+2 \mu \phi_{j}^{\prime}(1) \phi_{k}^{\prime}(1)\right]
\end{aligned}
$$

are the mass and stiffness matrices for both systems, respectively.

The damping matrix for system 3 is

$$
\mathbf{C}:=\left[\alpha \int_{0}^{1} \phi_{j}^{\prime \prime}(s) \phi_{k}^{\prime \prime}(s) \mathrm{d} s+\beta \int_{0}^{1} \phi_{j}(s) \phi_{k}(s) \mathrm{d} s\right]
$$

while, for system 4 , it reads

$$
\mathbf{C}:=\left[\xi_{e} \phi_{j}(1) \phi_{k}(1)+\xi_{t} \phi_{j}^{\prime}(1) \phi_{k}^{\prime}(1)\right]
$$

\section{Appendix C: Exact Bifurcation Loci for System 3}

The actual configuration of the rectilinear beam in Fig. 5a is described by the transversal displacement field of the beam axis $u(s, t)$, where $s \in[0, \ell]$ is a curvilinear abscissa and $t$ is the time. The motion around the trivial configuration is governed by the following nondimensional equations and boundary conditions:

$$
\begin{gathered}
\ddot{u}+u^{\mathrm{IV}}+\alpha \dot{u}^{\mathrm{IV}}+\beta \dot{u}+2 \mu u^{\prime \prime}=0 \\
u_{A}=0 \\
u_{A}^{\prime}=0 \\
u_{B}^{\prime \prime}+\alpha \dot{u}_{B}^{\prime \prime}=0 \\
u_{B}^{\prime \prime \prime}+\alpha \dot{u}_{B}^{\prime \prime \prime}-v u_{B}=0
\end{gathered}
$$

where $\mu:=F \ell^{2} / 2 E I, \quad v:=K \ell^{3} / E I, \quad \alpha:=\eta \omega / E, \quad$ and $\beta:=$ $c \omega \ell^{4} / E I$ are nondimensional parameters and $\omega=\left(E I / m \ell^{4}\right)^{1 / 2}$ is a frequency; $\ell$ is the length of the beam, $E I$ is the bending stiffness, a dot denotes the time differentiation, the index $A$ evaluation is at $s=0$, and the index $B$ evaluation is at $s=\ell$.

By letting $u(s, t)=\phi(s) \exp (\lambda t)$, a differential eigenvalue problem follows:

$$
\begin{gathered}
(1+\alpha \lambda) \phi^{\mathrm{IV}}+2 \mu \phi^{\prime \prime}+\left(\lambda^{2}+\beta \lambda\right) \phi=0 \\
\phi_{A}=0 \\
\phi_{A}^{\prime}=0 \\
(1+\alpha \lambda) \phi_{B}^{\prime \prime}=0 \\
(1+\alpha \lambda) \phi_{B}^{\prime \prime \prime}-v \phi_{B}=0
\end{gathered}
$$

with $\lambda \in \mathbb{C}$ as the eigenvalue, and $\phi(s) \in \mathbb{C}$ as the associated (right) eigenvector. Equation ( $\underline{\mathrm{C} 2 \mathrm{a}})$ admits the solution

$$
\phi(s)=c_{1}[\cos (p s)-\cosh (q s)]+c_{2}\left[\sin (p s)-\frac{p}{q} \sinh (q s)\right]
$$

where

$$
\begin{aligned}
& q^{2}:=\frac{\sqrt{\mu^{2}-(1+\alpha \lambda)\left(\beta \lambda+\lambda^{2}\right)}-\mu}{(1+\alpha \lambda)} \\
& p^{2}:=\frac{\sqrt{\mu^{2}-(1+\alpha \lambda)\left(\beta \lambda+\lambda^{2}\right)}+\mu}{(1+\alpha \lambda)}
\end{aligned}
$$

Moreover, $\mathbf{c}:=\left(c_{1}, c_{2}\right)^{T}$ are arbitrary constants, and boundary conditions at $A$ have already been accounted for. It should be noted that factor $p / q$ has been introduced in Eq. (C3), in order it holds even when $q \rightarrow 0$.

By enforcing boundary conditions at $B$, two algebraic equations follow:

$$
\mathbf{S}_{\lambda} \mathbf{c}=\mathbf{0}
$$

where

$$
\mathbf{S}_{\lambda}:=\left[\begin{array}{cc}
-(1+\alpha \lambda)\left(p^{2} \cos (p)+q^{2} \cosh (q)\right) & -p(1+\alpha \lambda)(p \sin (p)+q \sinh (q)) \\
v(\cosh (q)-\cos (p))+ & -p(1+\alpha \lambda)\left(p^{2} \cos (p)+q^{2} \cosh (q)\right)+ \\
+p^{3}(1+\alpha \lambda)(\sin (p)-\sinh (q)) & -v \sin (p)+p v \sinh (q) / q
\end{array}\right]
$$




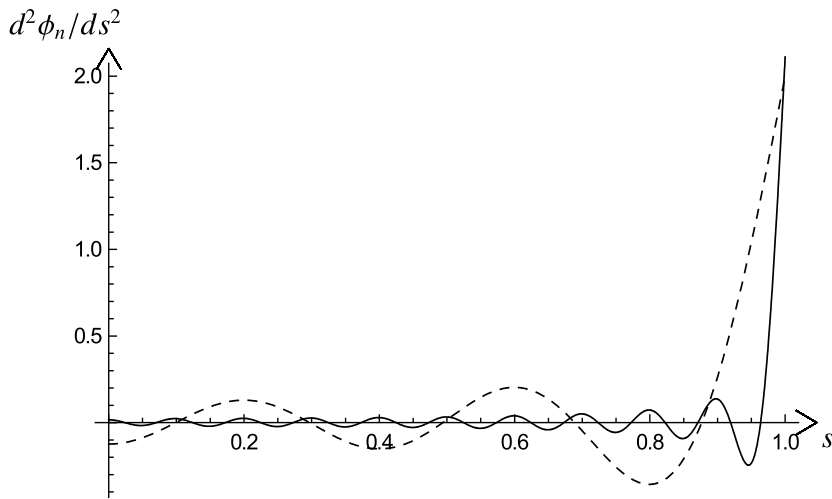

Fig. D1 Second derivative of the residue function for different discretizations (dashed line: five buckling eigenfunctions; solid line: 20 buckling eigenfunctions).

is the dynamic stiffness matrix of the system, depending on the eigenvalue $\lambda$. This matrix, however, also depends on the control parameters $(\mu, v)$ and the auxiliary parameters $(\alpha, \beta)$; that is, $\mathbf{S}_{\lambda}=\mathbf{S}_{\lambda}(\mu, v ; \alpha, \beta)$.

The characteristic equation supplies the eigenvalues as roots of $\operatorname{det} \mathbf{S}_{\lambda}(\mu, \nu ; \alpha, \beta)=0$. To restate the problem in real variables, the eigenvalues are written as $\lambda=\zeta+i \omega$, with $\zeta, \omega \in \mathbb{R}$, and then the characteristic equation is rewritten in the form

$$
f(\zeta, \omega ; \mu, \nu ; \alpha, \beta)+i g(\zeta, \omega ; \mu, \nu ; \alpha, \beta)=0
$$

with $f, g \in \mathbb{R}$. For a fixed set of parameter $(\mu, \nu ; \alpha, \beta)^{T}$, the system of two real equations $f=0, g=0$ furnishes the unknowns $\zeta, \omega$.

Hopf bifurcation occurs at the manifold $\mathcal{H}$ on which $\zeta=0, \omega \neq 0$, defined by

$$
\left\{\begin{array}{l}
f(0, \omega ; \mu, \nu ; \alpha, \beta)=0 \\
g(0, \omega ; \mu, \nu ; \alpha, \beta)=0
\end{array}\right.
$$

These equations, for a given pair of damping coefficients $(\alpha, \beta)$, implicitly define a multibranch curve $\mathcal{H}$ in the $(\nu, \mu)$ plane, parameterized by the $\omega$ parameter. No closed-form solutions (only numerical) can be pursued for Eqs. (C8).

Divergence occurs at locus $\mathcal{D}$ on which $f(0,0 ; \mu, \nu ; \alpha, \beta)=0$ and $g(0,0 ; \mu, v ; \alpha, \beta)=0$. Since $g$ is found to vanish identically, and $f$ is found to be independent of the damping coefficients, $\mathcal{D}$ is implicitly defined by $f(0,0 ; \mu, v ; 0,0)=0$.

\section{Appendix D: Discussion on Shape Functions Used for System 4}

Motivations for adopting the shape functions in Eq. (31) for Galerkin discretization of system 4 are discussed here.

1) Since the buckling eigenfunction equation (31a) is not able to describe the bending moment at end $B$ caused by the torsional dashpot, it needs to enlarge the set of the shape functions by including an element for which the second derivative does not vanish at $B$ (e.g., a parabola)

2) If $\phi_{n}(s)=s^{2}$ were simply taken due to the fact that

$$
\lim _{n \rightarrow \infty} \sum_{k=1}^{n-1} c_{k} \phi_{k}(s)-s^{2}=0
$$

$s \in[0,1)$ [i.e., the series of eigenfunctions tends to $s^{2}$ when $n \rightarrow \infty$ (everywhere, except at $s=1$ )], large stiffness and mass matrices would be found to be nearly singular. Indeed, the nontrivial set of Lagrangian coordinates $q_{k}(t)=c_{k}(k=1,2, \ldots, n-1), q_{n}(t)=$ -1 describes the trivial motion

$$
u(s, t)=\sum_{k=1}^{n} q_{k}(t) \phi_{k}(s)=0
$$

To avoid this occurrence, $\phi_{n}(s)$ must be made orthogonal to the space of the first $n-1$ shape functions, as made in Eq. (31b). Figure D1 shows that, when $n \rightarrow \infty$, the second derivative of $\overline{\phi_{n}(s)}$ tends to the Dirac delta at $s=1$.

In closing, it is worth noticing that, since the mechanical behavior of the system is strongly dependent of the concentrated bending moment at $B$, if a discretization were performed not including a function $\phi_{n}(s)$, as in Eq. (31b), some critical points would have been lost and, consequently, a wrong scenario would have been obtained that was different from that of Fig. 7.

\section{Acknowledgment}

This work was supported by the Italian Ministry of University (MIUR) through a PRIN cofinanced program.

\section{References}

[1] Seyranian, A. P., and Mailybaev, A. A., Multiparameter Stability Theory with Mechanical Applications, World Scientific, Hackensack, NJ, 2004, pp. 21-148.

[2] Gryazina, E. N., and Polyak, B. T., "Stability Regions in the Parameter Space: D-Decomposition Revisited," Automatica, Vol. 42, No. 1, 2006, pp. 13-26. doi:10.1016/j.automatica.2005.08.010

[3] Gryazina, E. N., and Polyak, B. T., "Multidimensional Stability Domain of Special Polynomial Families," Automation and Remote Control (English Translation), Vol. 68, No. 12, 2007, pp. 2128-2141. doi:10.1134/S000511790712003X

[4] Guckenheimer, J., and Holmes, P., Nonlinear Oscillations, Dynamical Systems and Bifurcations of Vector Fields, Vol. AMS 42, SpringerVerlag, New York, 1983, pp. 117-165.

[5] Dobson, I., "Computing a Closest Bifurcation Instability in Multidimensional Parameter Space," Journal of Nonlinear Science, Vol. 3, No. 1, 1993, pp. 307-327. doi:10.1007/BF02429868

[6] Dobson, I., "Distance to Bifurcation in Multidimensional Parameter Space: Margin Sensitivity and Closest Bifurcations," Bifurcation Control: Theory and Applications, edited by G. Chen, D. J. Hill, and X. H. Yu, Lecture Notes in Control and Information Sciences, SpringerVerlag, Berlin, 2003, pp. 49-66.

[7] Luongo, A., "Eigensolutions Sensitivity for Nonsymmetric Matrices with Repeated Eigenvalues," AIAA Journal, Vol. 31, No. 7, 1993 , pp. 1321-1328. doi:10.2514/3.11770

[8] Nayfeh, A. H., and Balachandran, B., Applied Nonlinear Dynamics: Analytical, Computational, and Experimental Methods, Wiley, New York, 1995, pp. 423-460.

[9] Luongo, A., "Eigensolutions of perturbed nearly defective matrices," Journal of Sound and Vibration, Vol. 185, No. 3, 1995, pp. 377-395. doi:10.1006/jsvi.1995.0387

[10] Leipholz, M., Stability Theory, Academic Press, New York, 1970.

[11] Di Egidio, A., Luongo, A., and Paolone, A., "Linear and Non-Linear Interactions Between Static and Dynamic Bifurcations of Damped Planar Beams," International Journal of Non-Linear Mechanics, Vol. 42, No. 1, 2007, pp. 88-98. doi:10.1016/j.ijnonlinmec.2006.12.010

[12] Maddox, N. R., "On the Number of Modes Necessary for Accurate Response and Resulting Forces in Dynamic Analysis," Journal of Applied Mechanics, Vol. 42, No. 2, 1975, pp. 516-517. doi:10.1115/1.3423622

[13] Luongo, A., Paolone, A., and Di Egidio, A., "Sensitivities and Linear Stability Analysis Around a Double-Zero Eigenvalue," AIAA Journal, Vol. 38, No. 4, 2000, pp. 702-710. doi: $10.2514 / 2.1013$ 